\title{
CLASSIFICATION OF HOMOGENEOUS BOUNDED DOMAINS OF LOWER DIMENSION
}

\author{
SOJI KANEYUKI AND TADASHI TSUJI*)
}

\section{Introduction}

The theory of classification of homogeneous bounded domains in the complex number space $C^{n}$ has been developed mainly in the recent papers [10], [6], [3] and [7]. As a result, the classification is reduced to that of $S$-algebras due to Takeuchi [7] which correspond to irreducible Siegel domains of type I or type II (For the definition of irreducibility see $\S 1$ ). On the other hand Pjateckii-Sapiro [5] found large classes of homogeneous Siegel domains obtained from classical self-dual cones. Even in lowerdimensional cases, however, there are still homogeneous Siegel domains which do not appear in his results.

In this article, we give a method of classification of $S$-algebras which correspond to irreducible Siegel domains; applying this, we classify all irreducible Siegel domains of type I and of type II up to dimension 10 and 8 , respectively.

After reviewing results of [3] and [8] in $\S 1$, we define in $\S 2 \mathrm{~N}$ algebras of type II and establish a relation between $N$-algebras and $S$ algebras. In $\S 3$ we define skeletons of type I or type II and isomorphisms among them. It turns out that to each isomorphism class of $\mathrm{N}$-algebras there corresponds an isomorphism class of certain skeletons (Lemma 3.1 and Lemma 3.2). We classify all skeletons which are necessary to find all the $\mathrm{N}$-algebras corresponding to the above-mentioned Siegel domains (Prop. 3.5 and Prop. 3.6). In $\S 4$ we will first restrict our attention to 3-skeletons of type I and 2-skeletons of type II. We study how to construct $N$-algebras from such a skeleton (Lemma 4.1 and 4.6) and study under what conditions these $N$-algebras are isomorphic

Received January 9, 1973.

*) The first-named author is a Matsunaga Science fellow. This work was begun while the first-named author had been an ONR Postdoctoral Research Associate at Washington University, St. Louis, Mo. in 1969-70. 
(Lemma 4.2, 4.4, 4.5 and 4.8). In $\S 5$ and $\S 6$, applying results in $\S 4$, we will obtain all the $N$-algebras which correspond to each of skeletons classified in Proposition 3.5 and 3.6.

In $\S 7$, summing up results in $\S 5$ and $\S 6$ we get the main theorem (Theorem 7.1). We give also the numbers of irreducible Siegel domains in the respective dimensions (Theorem 7.2). Furthermore we give the explicit forms of all irreducible Siegel domains of type I (resp. type II) up to dimension 7 (resp. 8). Some of them are already known in Pjateckii-Sapiro [5]; but others are new and most of them are Siegel domains obtained from non-selfdual cones. It should be noted that there exists a unique one-parameter family of non-isomorphic irreducible Siegel domains of type II in $C^{7}$ or in $C^{8}$ (cf. $\S 6$ and $\S 7$ ).

Throughout this paper, we will employ notations and terminologies in the previous article [3]. We denote by $E_{n}$ the unit matrix of degree $n$ and by $O(n)$ the real orthogonal group of degree $n$.

\section{§ 1. Basic theorems and $N$-algebras of type I}

1.1. Vinberg, Gindikin and Pjateckii-Sapiro [10] proved that every homogeneous bounded domain $D$ is realized as a (affine) homogeneous Siegel domain of type I or type II. If $D$ is realized as that of type I, it is called of tube type. A homogeneous bounded domain is called irreducible, if it is not (holomorphically) isomorphic to a direct product of any two homogeneous bounded domains of lower dimension. Then the following theorems are known:

THEOREM A ([3]). Every homogeneous bounded domain $D$ is isomorphic to a direct product of irreducible domains; the decomposition is unique up to an order. Furthermore $D$ is of tube type if and only if each irreducible factor of $D$ is of tube type.

THEOREM B ([3], [4]). Let $D(V, F)$ and $D\left(V^{\prime}, F^{\prime}\right)$ be homogeneous Siegel domains of type I or type II. Then $D(V, F)$ is (holomorphically) isomorphic to $D\left(V^{\prime}, F^{\prime}\right)$ if and only if they are mutually linearly equivalent. In particular homogeneous Siegel domains $D(V)$ and $D\left(V^{\prime}\right)$ ' of type $I$ are mutually isomorphic if and only if the homogeneous convex cones $V$ and $V^{\prime}$ are linearly equivalent to each other.

A homogeneous convex cone $V$ is called irreducible if it is not linearly 
equivalent to a direct sum of any two homogeneous convex cones.

THEOREM C $C^{*)}$ ([3]). A homogeneous Siegel domain $D(V, F)$ of type $I$ or type II is irreducible as a homogeneous bounded domain if and only if the homogeneous cone $V$ is irreducible.

It is known in [3] that a homogeneous Siegel domain of type II can not be realized as a homogeneous Siegel domain of type I. Therefore, in view of the above theorems, what we need to do, in order to classify homogeneous bounded domains up to holomorphic equivalence, is

A) to classify irreducible homogeneous convex cones $V$ up to linear equivalence, and

B) to classify homogeneous Siegel domains $D(V, F)$ of type II with $V$ irreducible, up to linear equivalence.

1.2. We recall $N$-algebras of type I due to Vinberg [8].

DEFINITION ([8]). Let $N$ be a finite dimensional associative algebra over the real number field $\boldsymbol{R}$, and $m(\geq 2)$ be a positive integer. Suppose that $N$ is the direct sum of bigraded subspaces $N_{i j}(1 \leq i<j \leq m)$ and that $N$ is equipped with a positive definite inner product $\langle$,$\rangle . Then N$ is called an $N$-algebra of type $I$ of rank $m$, if the following conditions are satisfied;

(N1) $N_{i j} N_{j k} \subset N_{i k}$,

(N2) $N_{i j} N_{\ell k}=(0)$ if $j \neq \ell$,

(N3) $\left\langle N_{i j}, N_{k \ell}\right\rangle=0$ if $i \neq k$ or $j \neq \ell$,

(N4) for every $a_{i j} \in N_{i j}$ and $b_{j k} \in N_{j k}$,

$$
\left\langle a_{i j} b_{j k}, a_{i j} b_{j k}\right\rangle=\frac{1}{n_{j}}\left\langle a_{i j}, a_{i j}\right\rangle\left\langle b_{j k}, b_{j k}\right\rangle,
$$

where $n_{j}=1+\frac{1}{2} \sum_{s<j} \operatorname{dim} N_{s j}+\frac{1}{2} \sum_{s>j} \operatorname{dim} N_{j s}$,

(N5) if $a_{i k} \in N_{i k}, b_{j k} \in N_{j k}(i<j)$ and

$\left\langle a_{i k}, N b_{j k}\right\rangle=0$, then $\left\langle N a_{i k}, N b_{j k}\right\rangle=0$.

An $N$-algebra of type I of rank one is defined to be an empty set. (N4) is equivalent to the following $\left(\mathrm{N}^{\prime}\right)$ or $\left(\mathrm{N} 4^{\prime \prime}\right)$.

*) Taking this opportunity we correct a small error in the proof of this theorem; that is, in the line $6 \mathrm{ff}$, page $126[3],\left(\Delta^{(\alpha)}-\Delta^{(\beta)}+\Delta^{(\mu)}\right) / 2$ and $\left(\Delta^{(\mu)}-\Delta^{(\nu)}+\Delta^{(\alpha)}\right) / 2$ are not roots only for $\alpha \neq \beta$ or $\mu \neq \nu$. But, since we have $\left[j r_{\alpha}, W_{2}\right]=\left[j r_{\mu}, W_{1}\right]=0$, the equality $\left[j R_{1}, W_{2}\right]=\left[j R_{2}, W_{1}\right]=0$ in the line $7 \mathrm{ff}$ is still valid. 
$\left(\mathrm{N} 4^{\prime}\right)$ For every $a_{i j}, a_{i j}^{\prime} \in N_{i j}$ and $b_{j k}, b_{j k}^{\prime} \in N_{j k}$,

$$
\left\langle a_{i j} b_{j k}, a_{i j}^{\prime} b_{j k}^{\prime}\right\rangle+\left\langle a_{i j} b_{j k}^{\prime}, a_{i j}^{\prime} b_{j k}\right\rangle=\frac{2}{n_{j}}\left\langle a_{i j}, a_{i j}^{\prime}\right\rangle\left\langle b_{j k}, b_{j k}^{\prime}\right\rangle .
$$

$\left(\mathrm{N}^{\prime \prime}\right)$ For orthonormal bases $\left\{e_{i j}^{\ell}\right\}$ of $N_{i j}$,

$$
\left\langle e_{i j}^{a} e_{j k}^{b}, e_{i j}^{c} e_{j k}^{d}\right\rangle+\left\langle e_{i j}^{a} e_{j k}^{a}, e_{i j}^{c} e_{j k}^{b}\right\rangle=\frac{2}{n_{j}} \delta_{a c} \delta_{b d}
$$

Let $N=\sum_{i<j} N_{i j}$ be an $N$-algebra of type I of rank $m$. A permutation $\sigma$ of the index set $\{1,2, \cdots, m\}$ is called admissible to $N$ if $N_{i j}=$ (0) as long as $i<j$ and $\sigma(i)>\sigma(j)$. Let $\sigma$ be a permutation admissible to $N$. If we replace each index $i$ by $\sigma(i)$ in $N$, then we get a new $N$ algebra $N^{\sigma}$ of type I different from $N$ only in bigrading. Let $N$ and $N^{\prime}$ be two $N$-algebras of type I of rank $m$. Then $N$ is said to be isomorphic to $N^{\prime}$ if there exist a permutation $\sigma$ admissible to $N$ and an algebra isomorphism $f$ of $N^{\sigma}$ onto $N^{\prime}$ which is not only bigrade-preserving but isometric relative to the respective inner products. It is known in Vinberg [8] that there exists a natural bijection between the set of all linear equivalence classes of homogeneous convex cones and the set of all isomorphism classes of $N$-algebras of type I. To the $N$-algebra of type I of rank one there corresponds the cone of the positive real halfline.

Thus, to solve the problem A) we have only to consider $N$-algebras of type $\mathrm{I}$.

\section{§ 2. $N$-algebras of type II}

We shall begin with some definitions due to Vinberg [8]. Let $m$ be a positive integer and $\mathfrak{A}$ be a finite dimensional algebra over $\boldsymbol{R}$. Then $\mathfrak{O}$ is called a matrix algebra of rank $m+1$ if it is bigraded with subspaces $\mathfrak{A}_{i j}(1 \leq i, j \leq m+1)$ such that $\mathfrak{A}_{i j} \mathfrak{U}_{j k} \subset \mathfrak{A}_{i k}, \mathfrak{U}_{i i} \neq(0)(1 \leq i \leq m$ +1 ) and that $\mathfrak{A}_{i j} \mathfrak{A}_{k \ell}=(0)$ for $j \neq k$. Let $\mathfrak{U}=\sum_{1 \leq i, j \leq m+1} \mathfrak{A}_{i j}$ be a matrix algebra of rank $m+1$. By an involution of $\mathfrak{A}$ we mean an involutive anti-automorphism $*$ of $\mathfrak{A}$ such that

$$
\mathfrak{A}_{i j}^{*}=\mathfrak{A}_{j i} \quad(1 \leq i, j \leq m+1) .
$$

A complex structure $j$ of a matrix algebra $\mathfrak{U}=\sum_{1 \leq i, j \leq m+1} \mathfrak{H}_{i j}$ of rank $m+1$ with an involution $*$ is, by definition, a linear endomorphism of 
the subspace $\sum_{1 \leq i \leq m}\left(\mathfrak{U}_{i, m+1}+\mathfrak{U}_{m+1, i}\right)$ of $\mathfrak{U}$ such that

$$
\begin{gathered}
j \mathfrak{U}_{i, m+1}=\mathfrak{A}_{i, m+1} \quad(1 \leq i \leq m), \\
j \circ *=* \circ j, \\
j^{2}=-1 .
\end{gathered}
$$

We note that (2.1), (2.2) and (2.3) imply that $j \mathfrak{A}_{m+1, i}=\mathfrak{A}_{m+1, i}(1 \leq i \leq m)$. From now on we shall use the following notations (cf. [8]);

$$
\begin{array}{ll}
{[a, b]=a b-b a} & (a, b \in \mathfrak{U}), \\
{[a, b, c]=a(b c)-(a b) c} & (a, b, c \in \mathfrak{U}), \\
n_{i j}=\operatorname{dim} \mathfrak{U}_{i j} & (1 \leq i, j \leq m+1), \\
n_{i}=1+\frac{1}{2} \sum_{k<i} n_{k i}+\frac{1}{2} \sum_{i<k} n_{i k},
\end{array}
$$

and we will denote by $a_{i j}$ the $\mathfrak{A}_{i j}$-component of an element $a \in \mathfrak{A}$. In what follows, we will consider exclusively $S$-algebras (cf. Takeuchi [7]) with the additional condition $(T, 0)$, which we call $T$-algebras of type II in accordance with the usual $T$-algebras in Vinberg [8].

DEFINITION 2.1 (cf. [7]). Let $\mathfrak{A}=\sum_{1 \leq i, j \leq m+1} \mathfrak{A}_{i j}$ be a matrix algebra of rank $m+1$ with an involution $*$ and a complex structure $j$. Then the triple $(\mathfrak{H}, *, j)$ is called a $T$-algebra of type $I I$ of rank $m$ if the following axioms are satisfied;

(T. 0) $\mathfrak{A}_{i, m+1} \neq(0)$ for some $i(1 \leq i \leq m)$,

(T. 1) Each subalgebra $\mathfrak{U}_{i i}(1 \leq i \leq m+1)$ is isomorphic to the algebra $\boldsymbol{R}$; These isomorphisms are denoted by $\rho$,

(T. 2) $a_{i i} b_{i j}=\rho\left(a_{i i}\right) b_{i j}, a_{i j} b_{j j}=\rho\left(b_{j j}\right) a_{i j}(1 \leq i, j \leq m+1)$,

(T. 3) $\operatorname{Sp}[a, b]=0(a, b \in \mathfrak{U})$, where $\mathrm{Sp}$ is defined by $\operatorname{Sp} a=\sum_{1 \leq i \leq m+1} n_{i} \rho\left(a_{i i}\right)$,

(T. 4) $\operatorname{Sp}[a, b, c]=0 \quad(a, b, c \in \mathfrak{U})$,

(T. 5) $\operatorname{Sp} a a^{*}>0$ if $a \neq 0(a \in \mathfrak{U})$,

(T. 6) $[a, b, c]=0 \quad\left(a, b, c \in \sum_{1 \leq i \leq j \leq m+1} \mathfrak{A}_{i j}\right)$,

(T. 7) $\left[a, b, b^{*}\right]=0 \quad\left(a, b \in \sum_{1 \leq i \leq j \leq m+1} \mathfrak{F}_{i j}\right)$,

(T. 8) $j\left(a_{i j} b_{j, m+1}\right)=a_{i j} j\left(b_{j, m+1}\right) \quad(1 \leq i<j \leq m)$,

(T. 9) $\quad \operatorname{Sp}(j a j b)=\operatorname{Sp} a b \quad\left(a, b \in \sum_{1 \leq i \leq m}\left(\mathfrak{A}_{i, m+1}+\mathfrak{A}_{m+1, i}\right)\right)$.

Remark 2.2. (T. 1)-(T. 7) imply that a T-algebra of type II of rank $m$ is itself a usual $T$-algebra of rank $m+1$ (cf. [8]). 
DEFINITION 2.3. Let $N=\sum_{1 \leq i<j \leq m+1} N_{i j}$ be an $N$-algebra of type I of rank $m+1$ with the inner product $\langle$,$\rangle and j$ be a linear endomorphism of the subspace $\sum_{1 \leq i \leq m} N_{i, m+1}$ of $N$. Then the triple $(N,\langle\rangle, j$,$) is$ called an $N$-algebra of type II of rank $m$ if the following conditions are satisfied;

$$
\begin{gathered}
N_{i, m+1} \neq(0) \quad \text { for some } i(1 \leq i \leq m), \\
j N_{i, m+1}=N_{i, m+1} \quad(1 \leq i \leq m), \\
j^{2}=-1, \\
\langle j a, j b\rangle=\langle a, b\rangle \quad\left(a, b \in \sum_{1 \leq i \leq m} N_{i, m+1}\right), \\
j(a b)=a j(b) \quad\left(a \in \sum_{1 \leq i<j \leq m} N_{i j,} b \in \sum_{1 \leq i \leq m} N_{i, m+1}\right) .
\end{gathered}
$$

The above $j$ is called the complex structure of $N$. For simplicity we will often denote $(\mathfrak{A}, *, j)$ by $\mathfrak{A}$ and $(N,\langle\rangle, j$,$) by N$, respectively.

Let $\mathfrak{U}$ be a $T$-algebra of type II of rank $m$ and $N$ be an $N$-algebra of type II of rank $m$. Then a permutation $\sigma$ of the index set $\{1,2, \cdots$, $m+1\}$ is said to be admissible to $\mathfrak{A}$ (resp. $N)$ if $\sigma(m+1)=m+1$ and if $\mathfrak{U}_{i j}=0$ (resp. $N_{i j}=0$ ) as long as $i<j$ and $\sigma(i)>\sigma(j)$. For a permutation $\sigma$ admissible to $\mathfrak{U}\left(\right.$ resp. $N$ ), we have a new $T$-algebra $\mathfrak{U}^{\sigma}$ (resp. an $N$-algebra $N^{\circ}$ ) of type II of rank $m$ by replacing each index $i$ by $\sigma(i)$ in $\mathfrak{U}($ resp. $N$ ), which is different from $\mathfrak{A}($ resp. $N$ ) only in bigrading.

Definition $2.4([7])$. Let $(\mathfrak{X}, *, j)$ and $\left(\mathfrak{X}^{\prime}, *^{\prime}, j^{\prime}\right)$ be two $T$-algebras of type II of rank $m$. Then $\mathfrak{A}$ is said to be isomorphic to $\mathfrak{X}^{\prime}$ if there exist a permutation $\sigma$ admissible to $\mathfrak{A}$ and a grade-preserving algebra isomorphism $\varphi$ of $\mathfrak{U}^{\sigma}$ onto $\mathfrak{U}^{\prime}$ such that

$$
\begin{gathered}
\varphi \circ *=*^{\prime} \circ \varphi, \\
\varphi \circ j=j^{\prime} \circ \varphi \text { on } \sum_{1 \leq i \leq m}\left(\mathfrak{A}_{i, m+1}^{o}+\mathfrak{A}_{m+1, i}^{o}\right) .
\end{gathered}
$$

DEFINITION 2.5. Let $(N,\langle\rangle, j$,$) and \left(N^{\prime},\langle,\rangle^{\prime}, j^{\prime}\right)$ be two $N$-algebras of type II of rank $m$. Then $N$ is said to be isomorphic to $N^{\prime}$ if there exist a permutation $\sigma$ admissible to $N$ and a grade-preserving algebra isomorphism $\psi$ of $N^{o}$ onto $N^{\prime}$ such that

$$
\psi \text { is an isometry with respect to }\langle,\rangle \text { and }\langle,\rangle^{\prime} \text {, }
$$




$$
\psi \circ j=j^{\prime} \circ \psi \quad \text { on } \quad \sum_{1 \leq i \leq m} N_{i, m+1}^{o} \text {. }
$$

Let $(\mathfrak{A}, *, j)$ be a $T$-algebra of type II of rank $m$. We define the inner product $\langle$,$\rangle in \mathfrak{A}$ by putting

$$
\langle a, b\rangle=\operatorname{Sp} a b^{*}
$$

for $a, b \in \mathfrak{A}$ (cf. (T. 5) in Definition 2.1). Then this inner product has the following relations;

$$
\begin{gathered}
\left\langle a^{*}, b^{*}\right\rangle=\langle a, b\rangle, \quad\left\langle a b^{*}, c\right\rangle=\left\langle b a^{*}, c^{*}\right\rangle=\langle c b, a\rangle, \\
\left\langle a^{*} b, c\right\rangle=\left\langle b^{*} a, c^{*}\right\rangle=\langle a c, b\rangle
\end{gathered}
$$

for $a, b, c \in \sum_{1 \leq i<j \leq m+1} \mathfrak{A}_{i j}$ (cf. [8], p. 349, (46), (51), (52)). Let us put $N(\mathfrak{U})$ $=\sum_{1 \leq i<j \leq m+1} \mathfrak{A}_{i j}$. Then, as is known in Vinberg [8], $N(\mathfrak{U})$ is an $N$-algebra of type I of rank $m+1$ with respect to the inner product $\langle$,$\rangle , since$ ( $(\mathfrak{H}, *$ ) is a $T$-algebra of rank $m+1$ (cf. Remark 2.2). From (T. 8) and (T. 9), it follows that the above inner product $\langle$,$\rangle and the complex$ structure $j$ restricted to $\sum_{1 \leq i \leq m} \mathfrak{A}_{i, m+1}$ satisfy (2.6)-(2.9). Thus $N(\mathfrak{2})$ is an $N$-algebra of type II of rank $m$. We denote by $\Phi$ the mapping which assigns each $T$-algebra $(\mathfrak{U}, *, j)$ of type II of rank $m$ to the $N$-algebra $(N(\mathfrak{U}),\langle\rangle, j$,$) of type II of rank m$.

THEOREM 2.6. The mapping $\Phi$ induces a natural bijection $\tilde{\Phi}$ between the set of all isomorphism classes of T-algebras of type II of rank $m$ and the set of all isomorphism classes of N-algebras of type II of rank $m$.

Proof. We define $\tilde{\Phi}$ to be the mapping which carries the isomorphism class of $(\mathfrak{A}, *, j)$ to that of $(N(\mathfrak{U}),\langle\rangle, j$,$) . First we will show that$ $\tilde{\Phi}$ is well-defined and injective. Let $(\mathfrak{A}, *, j)$ and $\left(\mathfrak{U}^{\prime}, *^{\prime}, j^{\prime}\right)$ be two $T$ algebras of type II of rank $m$ and suppose that $(\mathfrak{H}, *, j)$ is isomorphic to $\left(\mathfrak{U}^{\prime}, *^{\prime}, j^{\prime}\right)$. Then there exist a permutation $\sigma$ admissible to $\mathfrak{A}$ and an isomorphism $\varphi$ of $\mathfrak{U}^{\sigma}$ onto $\mathfrak{U}^{\prime}$. Since $\varphi\left(\mathfrak{U}_{i j}\right)=\mathfrak{U}_{\sigma(i) \sigma(j)}^{\prime}(1 \leq i<j \leq m+1)$ and since $n_{i j}=0$ as long as $i<j, \sigma(i)>\sigma(j)$, we have $n_{i}=n_{\sigma(i)}^{\prime}$. Hence, it follows that for $a_{i j}, b_{i j} \in \mathfrak{A}_{i j}(1 \leq i<j \leq m+1)$

$$
\begin{aligned}
\left\langle\varphi\left(a_{i j}\right), \varphi\left(b_{i j}\right)\right\rangle^{\prime} & =\operatorname{Sp}\left(\varphi\left(a_{i j}\right) \varphi\left(b_{i j}\right)^{*^{\prime}}\right)=n_{\sigma(i)}^{\prime} \rho^{\prime}\left(\varphi\left(a_{i j} b_{i j}^{*}\right)\right) \\
& =n_{i} \rho\left(a_{i j} b_{i j}^{*}\right)=\left\langle a_{i j}, b_{i j}\right\rangle,
\end{aligned}
$$

where $\rho^{\prime}$ is the algebra isomorphism of $\mathfrak{H}_{i i}^{\prime}$ onto $\boldsymbol{R}$. Therefore $\varphi$ induces an isometry of $N(\mathfrak{U})^{\sigma}$ onto $N\left(\mathfrak{U}^{\prime}\right)$. From this and the Definition 2.4 and 
2.5, it can be seen that $\varphi$ induces an isomorphism of $\left(N(\mathfrak{U})^{\sigma},\langle\rangle, j,\right)$ onto $\left(N\left(\mathfrak{Q}^{\prime}\right),\langle,\rangle^{\prime}, j^{\prime}\right)$, which proves that $\tilde{\Phi}$ is well-defined.

Suppose that $(N(\mathfrak{V}),\langle\rangle, j$,$) is isomorphic to \left(N\left(\mathfrak{U}^{\prime}\right),\langle,\rangle^{\prime}, j^{\prime}\right)$. Then there exists a permutation $\sigma$ admissible to $N(\mathfrak{H})$ and a grade-preserving isomorphism $\psi$ of $N(\mathfrak{U})^{\sigma}$ onto $N\left(\mathfrak{U}^{\prime}\right)$. Let us define the map $\varphi$ of $\mathfrak{H}^{\sigma}$ onto $\mathfrak{U}^{\prime}$ as follows;

$$
\varphi= \begin{cases}\psi & \text { on } \mathfrak{A}_{i j}^{\sigma}, i<j, \\ \rho^{\prime-1} \circ \rho & \text { on } \mathfrak{A}_{i i}^{\sigma}, \\ *^{\prime} \circ \psi \circ * & \text { on } \mathfrak{A}_{i j}^{\sigma}, j<i .\end{cases}
$$

Then by using (2.12)-(2.15), we can show that $\varphi$ is an isomorphism of $\left(\mathfrak{A}^{\sigma}, *, j\right)$ onto $\left(\mathfrak{Q}^{\prime}, *^{\prime}, j^{\prime}\right)$, which implies that $\tilde{\Phi}$ is injective.

We want to show that $\tilde{\Phi}$ is surjective. Let $\left(N,\langle\rangle,, j_{1}\right)$ be an $N$ algebra of type II of rank $m$. Then by Vinberg [8], there exists a $T$ algebra $(\mathfrak{A}, *)$ of rank $m+1$ such that $N(\mathfrak{U})=N$ as $N$-algebras of type I. We define a complex structure $j$ on $\mathfrak{A}$ as follows;

$$
j=\left\{\begin{array}{lll}
j_{1} & \text { on } & \mathfrak{U}_{i, m+1}=N_{i, m+1} \\
* \circ j_{1} \circ * & \text { on } & \mathfrak{U}_{m+1, i}=N_{i, m+1}^{*}(1 \leq i \leq m) .
\end{array}\right.
$$

It remains for us to show that $(\mathfrak{H}, *, j)$ satisfies the axioms (T. 8) and (T. 9). (2.9) implies (T. 8). On the other hand, for $a_{i, m+1} \in \mathfrak{A}_{i, m+1}$, $b_{m+1, i} \in \mathfrak{A}_{m+1, i}(1 \leq i \leq m)$,

$$
\begin{aligned}
\operatorname{Sp}\left(j a_{i, m+1} j b_{m+1, i}\right) & =\left\langle j a_{i, m+1},\left(j b_{m+1, i}\right)^{*}\right\rangle=\left\langle j_{1} a_{i, m+1}, j_{1} b_{m+1, i}^{*}\right\rangle \\
& =\left\langle a_{i, m+1}, b_{m+1, i}^{*}\right\rangle=\operatorname{Sp}\left(a_{i, m+1} b_{m+1, i}\right),
\end{aligned}
$$

which implies (T. 9). So $\Phi((\mathscr{Y}, *, j))=\left(N,\langle\rangle,, j_{1}\right)$. q.e.d.

From the above theorem and Theorem A in Takeuchi [7], we get the following:

COROLLARY 2.7. There exists a bijection between the set of all isomorphism classes of $N$-algebras of type II and the set of all linear equivalence classes of homogeneous Siegel domains of type II.

Thus, to work out the problem B) in $\S 1$, we have only to consider $N$-algebras of type II.

\section{§ 3. Skeletons}

3.1. We will define an $m$-skeletons of type $I$. Let us put $m$ tiny 
circles on $\boldsymbol{R}^{2}$ so that they may form the vertices of a regular $m$-polygon; by a regular 1-polygon (resp. 2-polygon) we mean a point (resp. a line segment). Let us number these circles counterclockwise, starting from the vertex at the upper left corner. The $i$-th circle is called the vertex $i$, or simply $i$. Some of these circles may be joined by line segments. By the notation $i \sim j$ (resp. $i \not j$ ) we mean that the vertices $i$ and $j$ are joined (resp. not joined) by a line segment. The following assumption (*) has to be satisfied; (*) if $i<j<k, i \sim j$ and $j \sim k$, then $i \sim k$. A figure $S$ satisfying (*) is called an $m$-skeleton of type $I$, if a positive integer $n_{i j}$ is attached to each line segment $\overline{i j}(i<j)$ in $S$ in such a way that

S1) if $i<j<k, i \sim j$ and $j \sim k$, then $\max \left(n_{i j}, n_{j k}\right) \leq n_{i k}$,

S2) if $i<j<k<\ell, i \sim j, j \sim \ell, i \sim k, k \sim \ell, i \sim \ell$ and $j \neq k$, then $\max \left(n_{i j}+n_{i k}, n_{i j}+n_{k \ell}, n_{j \ell}+n_{i k}, n_{j \ell}+n_{k \ell}\right) \leq n_{i \ell}$.

We often denote the skeleton $S$ by the pair $\left(S,\left(n_{i j}\right)\right)$. An $m$-skeleton $S$ of type $\mathrm{I}$ is called connected if for any two vertices $i$ and $j$ there exists a series of vertices $i=i_{0}, i_{1}, \cdots, i_{s-1}, i_{s}=j$ such that $i_{k-1} \sim i_{k}$ for each $1 \leq k \leq s$.

Definition 3.1. Let $\left(S,\left(n_{i j}\right)\right)$ and $\left(S^{\prime},\left(n_{i j}^{\prime}\right)\right)$ be two $m$-skeletons of type I. $\mathrm{S}$ is said to be isomorphic to $S^{\prime}$, if there exists a permutation $\sigma$ of the set $\{1,2, \cdots, m\}$ such that

i) if $i<j$ and $\sigma(i)>\sigma(j)$ in $S$, then $i \not j$ in $S$,

ii) $\sigma(i) \sim \sigma(j)$ in $S^{\prime}$ if and only if $i \sim j$ in $S$,

iii) $n_{\sigma(i) \sigma(j)}^{\prime}=n_{i j}$.

It can be seen that the above isomorphism is an equivalence relation in the set of all $m$-skeletons of type I.

EXAMPLE. The following 5-skeletons of type $\mathrm{I}$ are connected and mutually isomorphic under the permutation $\sigma=\left(\begin{array}{lllll}1 & 2 & 3 & 4 & 5 \\ 3 & 2 & 1 & 5 & 4\end{array}\right)$. 

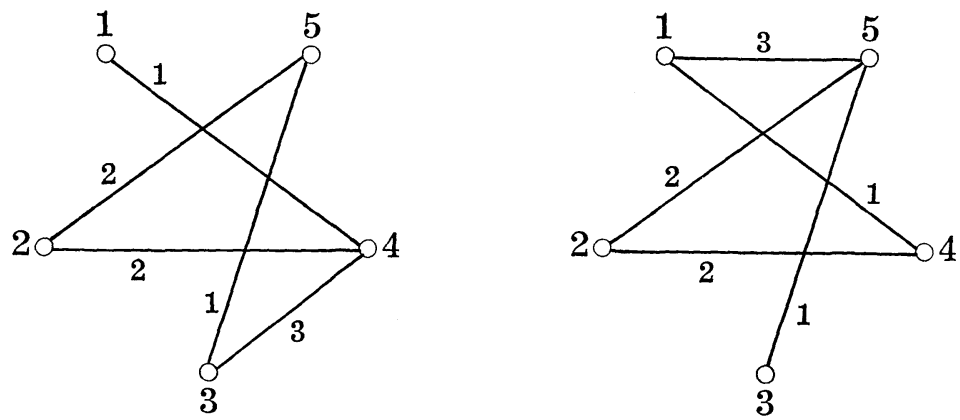

3.2. By an $m$-skeleton of type II we mean an $(m+1)$-skeleton $\left(\widetilde{S},\left(n_{i j}\right)\right)$ of type I satisfying the conditions;

i) there exists at least one vertex $i, 1 \leq i \leq m$ such that $i \sim m+1$; in this case each $n_{i, m+1}$ is an even number,

ii) only the last vertex $m+1$ is denoted by a black circle

An $m$-skeleton $\subseteq$ of type II is said to be connected if for any two vertices $i$ and $j(i, j \neq m+1)$ there exists a series of vertices $i=i_{0}, i_{1}$, $\cdots, i_{s-1}, i_{s}=j$ such that $1 \leq i_{1}, \cdots, i_{s-1} \leq m$ and that $i_{k-1} \sim i_{k}$ for each $1 \leq k \leq s$.

EXAMPLE. Consider the following 3-skeletons of type II.
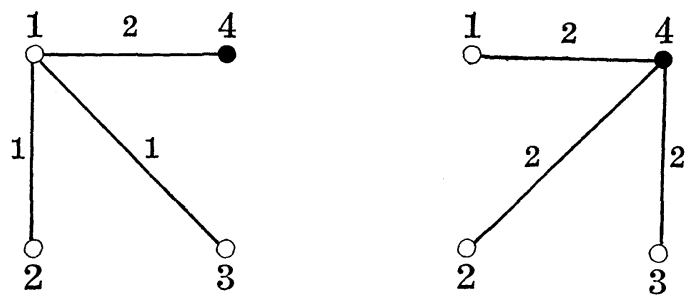

The first one is connected, while the second is not connected.

DEFINITION 3.2. Let $\left(\widetilde{S},\left(n_{i j}\right)\right)$ and $\left(\widetilde{\varsigma}^{\prime},\left(n_{i j}^{\prime}\right)\right)$ be two $m$-skeletons of type II. Then they are said to be isomorphic to each other, if there exists a permutation $\sigma$ of the set $\{1,2, \cdots, m, m+1\}$ leaving $m+1$ fixed and satisfying i)-iii) of Definition 3.1.

The above isomorphism is an equivalence relation in the set of all $m$-skeletons of type II.

3.3. Let $N=\sum_{i<j} N_{i j}$ be an $N$-algebra of type I of rank $m$. We put $n_{i j}=\operatorname{dim} N_{i j}$. For the $N$-algebra $N$ we define its diagram $S(N)$ in 
the following way*); at first $m$ tiny circles should be put and numbered in the same way as in 3.1 ; let us join the vertex $i$ with $j(i<j)$ by a line segment if and only if $n_{i j} \neq 0$, and let us attach the number $n_{i j}$ to each line segment $i \bar{j}$. For the case of $m=1, S(N)$ is defined to be just a vertex.

LEMMA 3.1. The diagram $S(N)$ of an $N$-algebra $N$ of type $I$ of rank $m$ is an $m$-skeleton of type I. If two $N$-algebras of type $I$ are isomorphic to each other, then so are their diagrams.

Proof. Suppose that three vertices $i<j<k$ in $S(N)$ satisfy $i \sim j$ and $j \sim k$. Let $x_{0}$ be a non-zero element in $N_{j k}$. Then, by (N4) the map of $N_{i j}$ to $N_{i k}$ defined by $x_{i j} \in N_{i j} \mapsto x_{i j} x_{0} \in N_{i k}$ is a linear isomorphism of $N_{i j}$ into $N_{i k}$. Hence we have $n_{i j} \leq n_{i k}$, and analogously $n_{j k} \leq n_{i k}$. $S(N)$ thus satisfies S1). Suppose that four vertices $i<j<k<\ell$ in $S(N)$ satisfy the conditions $i \sim j, j \sim \ell, i \sim k, k \sim \ell, i \sim \ell$ and $j \neq k$. Then, for arbitrary elements $x_{j \ell} \in N_{j \ell}$ and $x_{k \ell} \in N_{k \ell}$ we have $\left\langle x_{j \ell}, N x_{k \ell}\right\rangle=0$. Hence, by (N5) we have $\left\langle N N_{j \ell}, N N_{k \ell}\right\rangle=0$. Take non-zero elements $e_{j \ell} \in N_{j \ell}, e_{k \ell} \in N_{k \ell}$. Then the maps

$$
\begin{aligned}
& f: x_{i j} \in N_{i j} \longmapsto x_{i j} e_{j \ell} \\
& g: x_{i k} \in N_{i k} \longmapsto x_{i k} e_{k \ell}
\end{aligned}
$$

are linear isomorphisms of $N_{i j}$ into $N_{i \ell}$ and of $N_{i k}$ into $N_{i \ell}$, respectively (cf. (N4)). The condition $\left\langle N N_{j \ell}, N N_{k \ell}\right\rangle=0$ implies that the subspaces $f\left(N_{i j}\right)$ and $g\left(N_{i k}\right)$ of $N_{i \ell}$ are orthogonal to each other. Hence $n_{i j}+n_{i k}$ $\leq n_{i \ell}$. Other assertions in S2) are analogously proved. Thus $S(N)$ is an $m$-skeleton of type I. The second assertion of the lemma is immediate.

q.e.d.

Let $(N,\langle\rangle, j$,$) be an N$-algebra of type II of rank $m$. Then we can consider the diagram $S(N)$ of $N$ by regarding $N$ as an $N$-algebra of type I of rank $m+1$. By the diagram $\widetilde{S}(N)$ of $N$ as an $N$-algebra of type II of rank $m$ we mean the figure which is obtained from $S(N)$ by changing the color of the vertex $m+1$ in black. By the quite similar way as in Lemma 3.1 we get

Lemma 3.2. The diagram $\mathfrak{S}(N)$ of an $N$-algebra $(N,<\rangle, j$,$) of type$

*) To define the diagram of an $N$-algebra of type I was motivated by the diagram of a $T$-algebra due to Asano [1]. 
II of rank $m$ is an $m$-skeleton of type II. If two $N$-algebras of type II are isomorphic, then so are their diagrams.

3.4. Let $D(V, F)$ be a homogeneous Siegel domain of type II. Let $(N,\langle\rangle, j$,$) be the corresponding N$-algebra of type II and $\left(\widetilde{S},\left(n_{i j}\right)\right)$ be its diagram. Suppose that rank $N=m$. Then it follows from Takeuchi [7] that the figure which is obtained from $\left(\widetilde{S},\left(n_{i j}\right)\right)$ by removing the vertex $m+1$ and all line segments starting from $m+1$ is the diagram of the $N$-algebra of type I corresponding to the cone $V$. Hence, from Theorem $\mathrm{C}$ and a result of Asano [1] we have

Proposition 3.3. Let $D(V, F)$ be a homogeneous Siegel domain of type I or type II. Then it is irreducible if and only if the diagram of the $N$-algebra corresponding to $D(V, F)$ is connected.

Lemma 3.4. Let $D(V)$ (resp. $D(V, F)$ ) be an irreducible Siegel domain of type I (resp. type II). Let $N(V)$ (resp. $N(V, F)$ ) be the $N$-algebra corresponding to $D(V)$ (resp. $D(V, F)$ ). If $\operatorname{dim} D(V) \leq 10$, then $\operatorname{rank} N(V)$ $\leq 5$; if $\operatorname{dim} D(V, F) \leq 8$, then $\operatorname{rank} N(V, F) \leq 4$.

Proof. Suppose $\operatorname{rank} N(V)=n$ and $\operatorname{rank} N(V, F)=m$. Let $\left(S,\left(n_{i j}\right)\right)$ and $\left(\widetilde{S},\left(m_{i j}\right)\right)$ be the diagrams of $N(V)$ and $N(V, F)$, respectively. Note that $\operatorname{dim} D(V)=n+\sum_{1 \leq i<j \leq n} n_{i j}$ and $\operatorname{dim} D(V, F)=m+\sum_{1 \leq i<j \leq m} m_{i j}+$ $\sum_{1 \leq i \leq m} \frac{1}{2} m_{i, m+1}$ (cf. [8], [7]). Since $S$ and $\subseteq$ are connected by Proposition 3.3 , it follows from a result of Asano [1] that $\sum_{1 \leq i<j \leq n} n_{i j}>n-2$ and $\sum_{1 \leq i<j \leq m} m_{i j}>m-2$. So we get $n-2<\sum_{1 \leq i<j \leq n} n_{i j} \leq 10-n$. On the other hand, at least one $m_{i, m+1}$ is not zero and so $\sum_{i=1}^{m} \frac{1}{2} m_{i, m+1} \geq 1$. Hence $m-2<\sum_{1 \leq i<j \leq m} m_{i j} \leq 7-m$. Thus we have $n \leq 5$ and $m \leq 4$. q.e.d.

Thus, to solve the problem A) for the case of $\operatorname{dim} V \leq 10$ and $B$ ) for the case of $\operatorname{dim} D(V, F) \leq 8$ (cf. $\S 1$ ), our task is

I) to classify (up to isomorphism) all connected $n$-skeletons $\left(S,\left(n_{i j}\right)\right)$ of type I satisfying the condition

$$
\left\{\begin{array}{l}
n \leq 5 \\
\sum_{1 \leq i<j \leq n} n_{i j} \leq 10-n,
\end{array}\right.
$$

II) to classify all connected m-skeletons ( $\left(,\left(m_{i j}\right)\right)$ of type II satisfying the condition 


$$
\left\{\begin{array}{l}
m \leq 4 \\
\sum_{1 \leq i<j \leq m} m_{i j}+\sum_{1 \leq i \leq m} \frac{1}{2} m_{i, m+1} \leq 8-m,
\end{array}\right.
$$

III) for each skeleton $S$ or $\mathbb{S}$ obtained in I) or II) find (up to isomorphism) all the $N$-algebras whose diagrams are isomorphic to $S$ or $\mathbb{S}$.

The answers to the above problems I) and II) are given in the following two propositions, the proofs of which are quite elementary but tedious; so we may omit them.

Proposition 3.5. All the connected skeletons of type I satisfying (3.1) are (up to isomorphism) as follows;

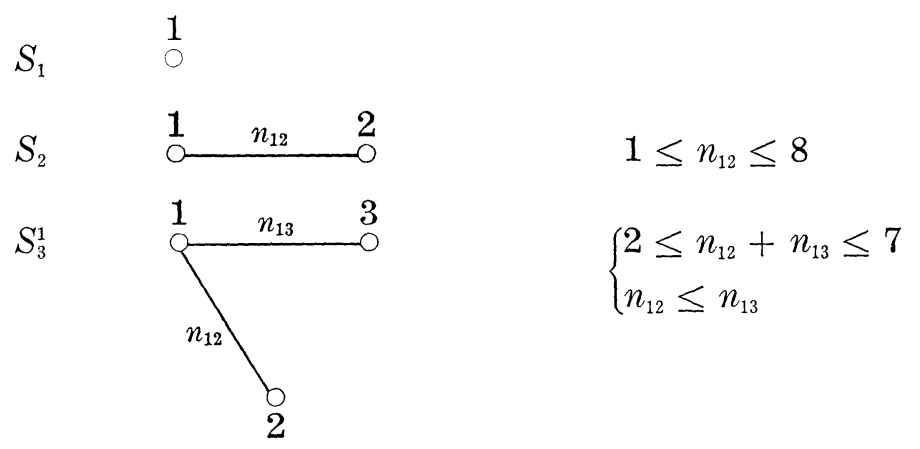

$S_{3}^{1^{*}}$

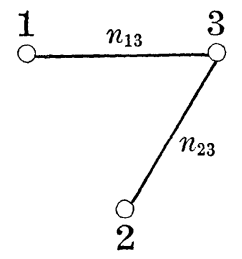

$$
\left\{\begin{array}{l}
2 \leq n_{13}+n_{23} \leq 7 \\
n_{23} \leq n_{13}
\end{array}\right.
$$

$S_{3}^{2}$

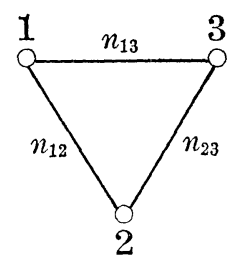

$$
\left\{\begin{array}{l}
3 \leq n_{12}+n_{23}+n_{13} \leq 7 \\
\operatorname{Max}\left(n_{12}, n_{23}\right) \leq n_{13}
\end{array}\right.
$$

$S_{\ddagger}^{1}$

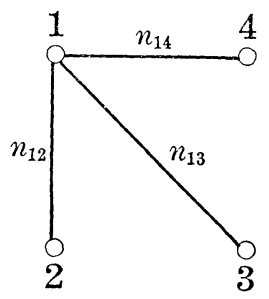

$$
\left\{\begin{array}{l}
3 \leq n_{12}+n_{13}+n_{14} \leq 6 \\
n_{12} \leq n_{13} \leq n_{14}
\end{array}\right.
$$


$S_{4}^{1 *}$

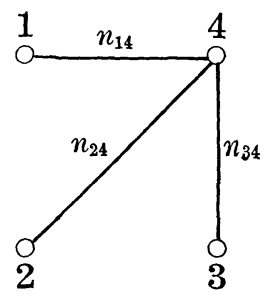

$$
\left\{\begin{array}{l}
3 \leq n_{14}+n_{24}+n_{34} \leq 6 \\
n_{34} \leq n_{24} \leq n_{14}
\end{array}\right.
$$

$S_{4}^{2}$

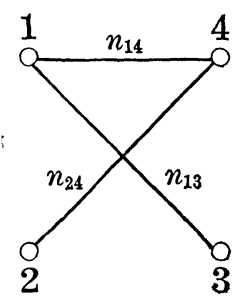

$$
3 \leq n_{13}+n_{24}+n_{14} \leq 6
$$

$S_{4}^{3}$

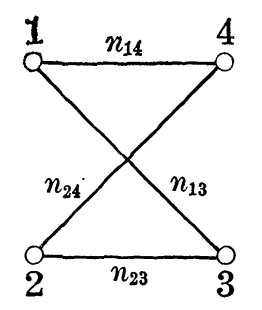

$$
\left\{\begin{array}{l}
4 \leq n_{13}+n_{14}+n_{23}+n_{24} \leq 6 \\
\operatorname{Max}\left(n_{13}, n_{23}, n_{24}\right) \leq n_{14}
\end{array}\right.
$$

$S_{4}^{4}$

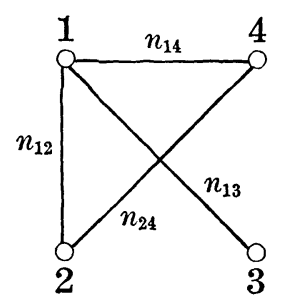

$$
\left\{\begin{array}{l}
4 \leq n_{12}+n_{13}+n_{24}+n_{14} \leq 6 \\
\operatorname{Max}\left(n_{12}, n_{24}\right) \leq n_{14}
\end{array}\right.
$$

$S_{4}^{4 *}$

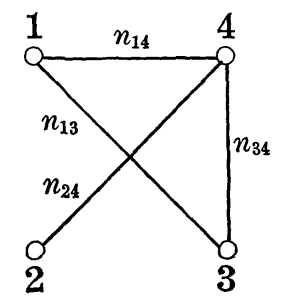

$$
\left\{\begin{array}{l}
4 \leq n_{13}+n_{14}+n_{24}+n_{34} \leq 6 \\
\operatorname{Max}\left(n_{13}, n_{34}\right) \leq n_{14}
\end{array}\right.
$$

$S_{4}^{5}$

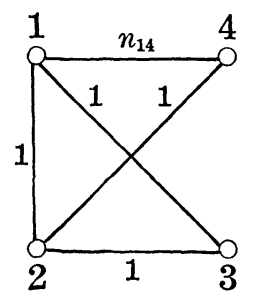

$$
n_{14}=1 \text { or } 2
$$


$S_{4}^{5 *}$

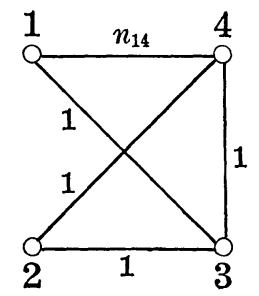

$S_{4}^{6}$

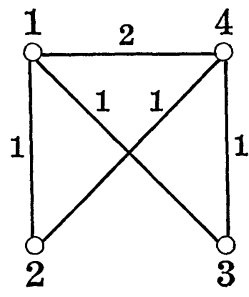

$S_{4}^{7}$

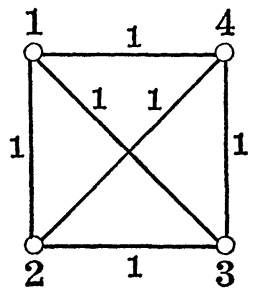

$S_{5}^{1}$

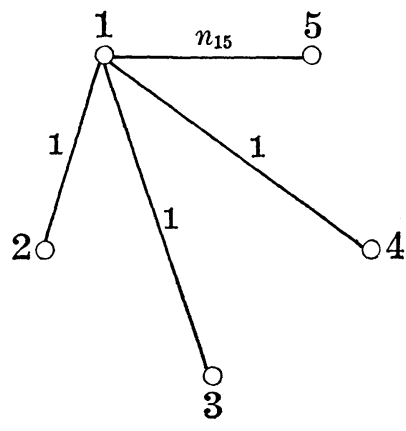

$S_{5}^{1 *}$

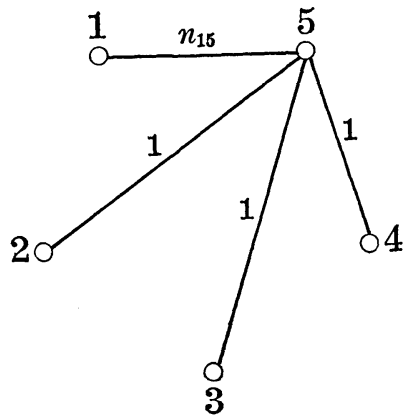

$n_{14}=1$ or 2

$$
n_{15}=1 \text { or } 2
$$

$n_{15}=1$ or 2 
$S_{5}^{2}$

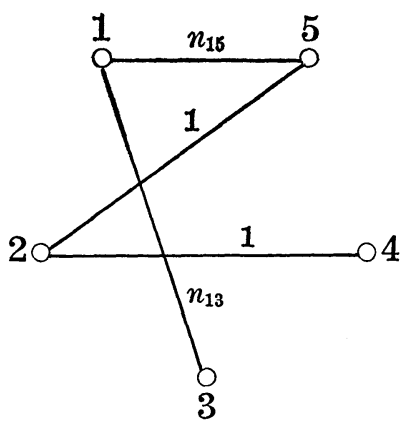

$S_{5}^{2 *}$

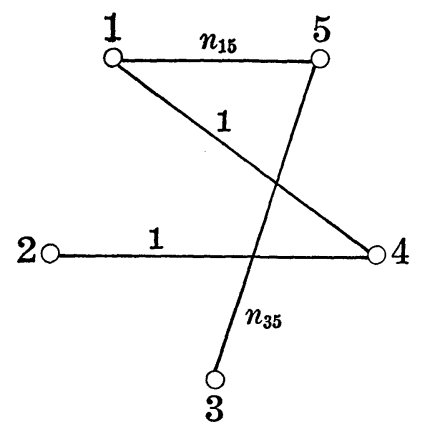

$S_{5}^{3}$

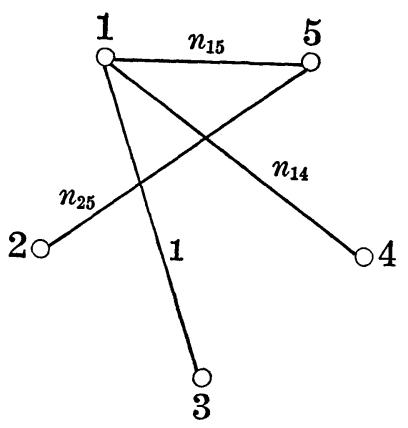

$S_{5}^{3 *}$

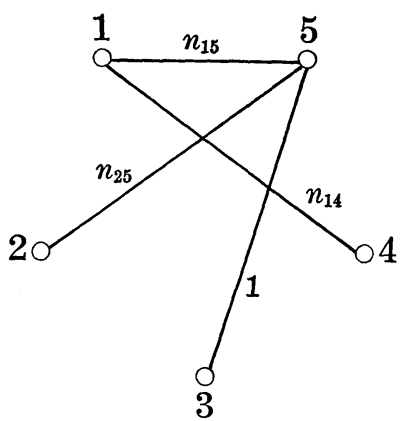

$2 \leq n_{13}+n_{15} \leq 3$

$2 \leq n_{35}+n_{15} \leq 3$

$3 \leq n_{14}+n_{15}+n_{25} \leq 4$

$3 \leq n_{14}+n_{15}+n_{25} \leq 4$ 
$S_{5}^{4}$

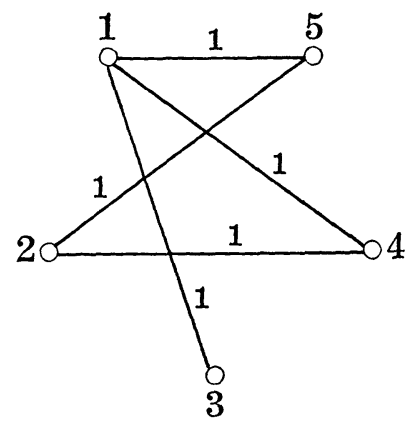

$S_{5}^{5}$

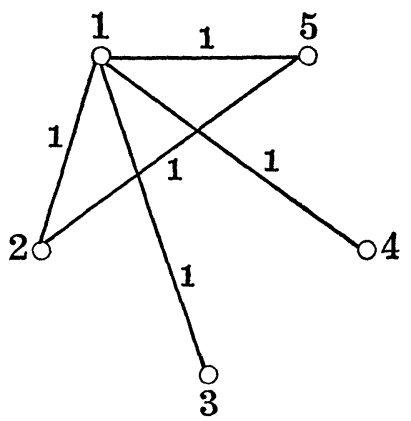

$S_{5}^{6}$

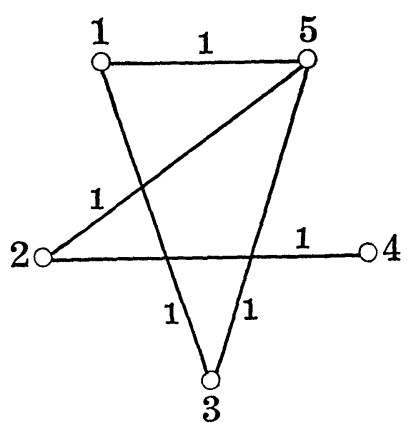

$S_{5}^{7}$

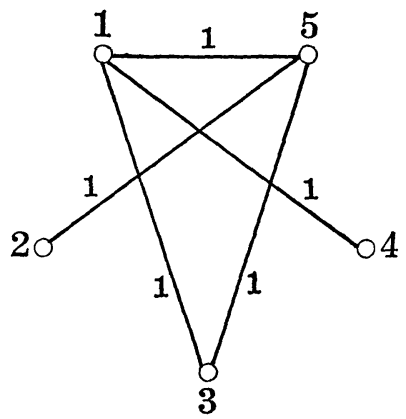

$S_{5}^{4 *}$

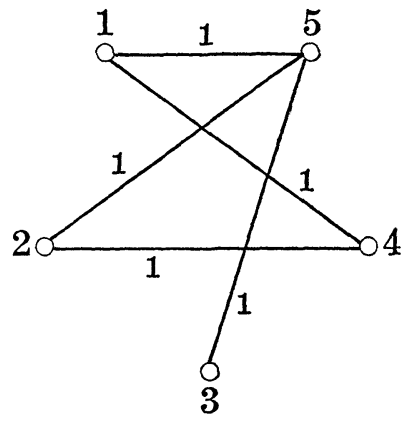

$S_{5}^{5^{*}}$

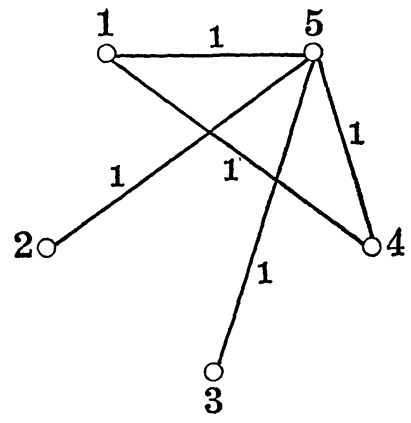

$S_{5}^{6 *}$

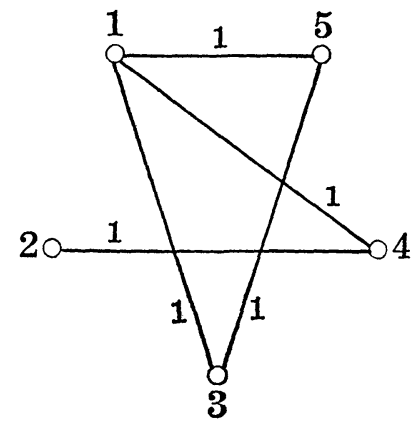


Proposition 3.6. All the connected skeletons of type II satisfying (3.2) are (up to isomorphism) as follows;

$\widetilde{S}_{1}$

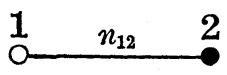

$\widetilde{S}_{2}^{1}$

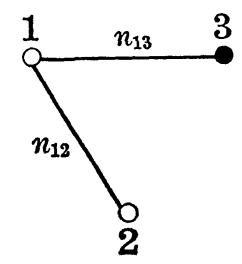

$\widetilde{S}_{2}^{2}$

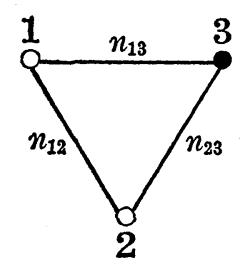

$\widetilde{S}_{3}^{1}$

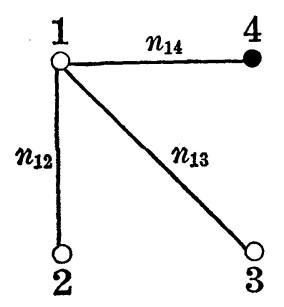

$\mathfrak{S}_{3}^{2}$

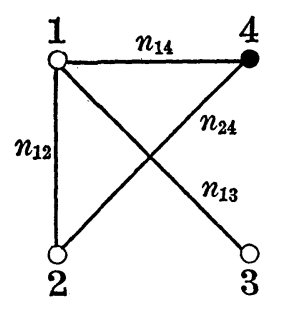

$\widetilde{S}_{3}^{3}$

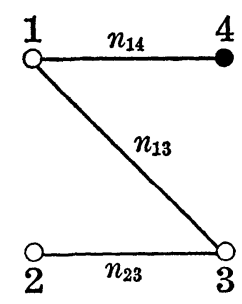

$1 \leq \frac{1}{2} n_{12} \leq 7$

$2 \leq n_{12}+\frac{1}{2} n_{13} \leq 6$.

$\left\{\begin{array}{l}3 \leq n_{12}+\frac{1}{2}\left(n_{23}+n_{13}\right) \leq 6 \\ \operatorname{Max}\left(n_{12}, n_{23}\right) \leq n_{13}\end{array}\right.$

$\left\{\begin{array}{l}3 \leq n_{12}+n_{13}+\frac{1}{2} n_{14} \leq 5 \\ n_{12} \leq n_{13}\end{array}\right.$

$$
3 \leq n_{13}+n_{23}+\frac{1}{2} n_{14} \leq 5
$$


$\Im_{3}^{4}$

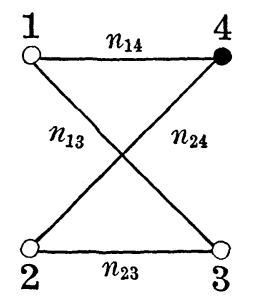

$$
\left\{\begin{array}{l}
\left(n_{13}, n_{23}, n_{14}, n_{24}\right)=(1,1,2,2), \\
(1,1,2,4),(2,1,2,2) .
\end{array}\right.
$$

$\Im_{3}^{5}$

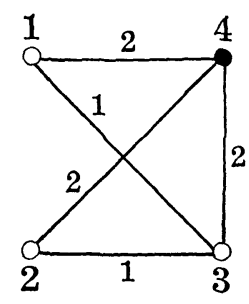

$\widetilde{\Im}_{3}^{6}$

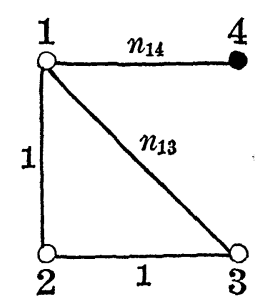

$$
\left(n_{13}, n_{14}\right)=(1,2),(1,4),(2,2) .
$$

$\varsigma_{3}^{7}$

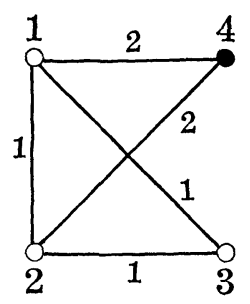

$\Im_{4}^{1}$

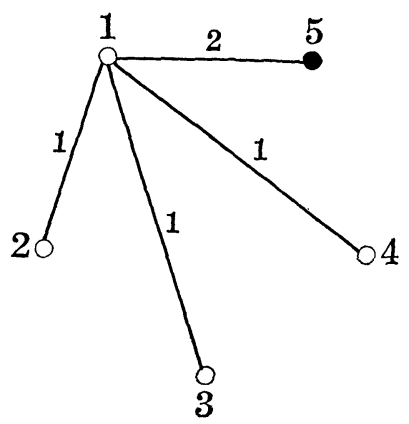

$\mathfrak{S}_{4}^{2}$

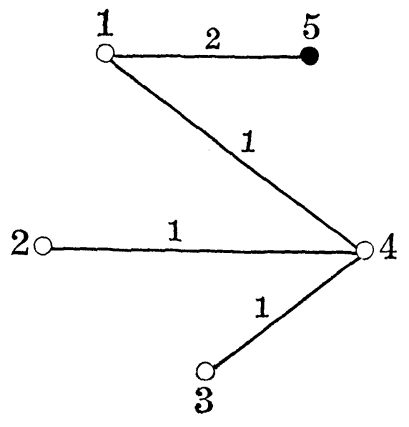


$\widetilde{\Im}_{4}^{3}$

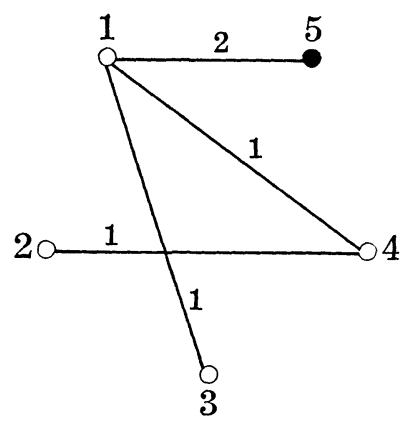

$\widetilde{\Im}_{4}^{4}$

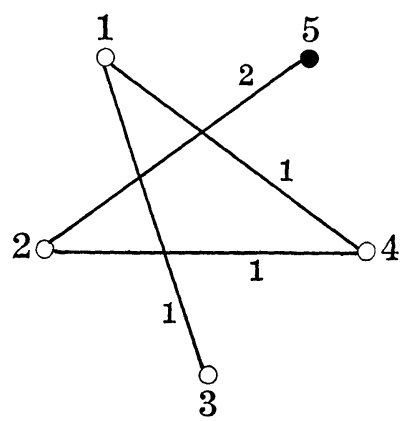

\section{§4. Some lemmas on $N$-algebras}

4.1. Let $S$ be the following 3-skeleton of type $I$

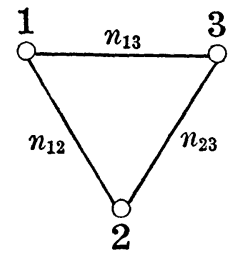

and we consider a real square matrix $A=\left(a_{(i j)(k \ell)}\right)$ of degree $n_{12} n_{23}$, where $1 \leq i, k \leq n_{12}, 1 \leq j, \ell \leq n_{23}$, (The double indices ( $i j$ ) should be put in the lexicographic order) satisfying the following conditions

$$
\begin{aligned}
& a_{(i j)(k \ell)}=a_{(k \ell)(i j)}, \\
& a_{(i j)(k \ell)}+a_{(i \ell)(k j)}=\frac{2}{n_{2}} \delta_{i k} \delta_{j \ell}, \\
& \quad \text { where } n_{2}=1+\frac{1}{2}\left(n_{12}+n_{23}\right),
\end{aligned}
$$

Such a matrix $A$ is not uniquely determined in general and may contain several parameters $t_{1}, \cdots, t_{s}$ which are indeterminate coefficients of $A$. So we write $A_{t}$ for $A$, where $t=\left(t_{1}, \cdots, t_{s}\right)$. The matrix $A_{t}$ is called the Grammian of $S$. Let $B$ be an $n_{12} n_{23} \times n_{13}$ real matrix and consider the matrix equation with $B$ as its indeterminate

$$
A_{t}=B^{t} B \text {. }
$$

It can be easily seen that the equation (4.4) has a solution if and only if $\operatorname{rank} A_{t} \leq n_{13}$. Let $B_{t}=\left(b_{i j}^{k}\right)$ be a solution of (4.4), where $1 \leq i \leq n_{12}$, 
$1 \leq j \leq n_{23}$, and $1 \leq k \leq n_{13}$. Let $N=N_{12}+N_{23}+N_{13}$ be the orthogonal direct sum of the euclidean vector spaces $N_{i j}$ with $\operatorname{dim} N_{i j}=n_{i j}$ $(1 \leq i<j \leq 3)$. Then, for fixed orthonormal bases $\left\{e_{i j}^{k}\right\}$ of $N_{i j}$ we define a multiplication in $N$ as follows:

$$
\begin{cases}e_{12}^{i} e_{23}^{j}=\sum_{k=1}^{n_{13}} b_{i j}^{k} e_{13}^{k}, & 1 \leq i \leq n_{12}, 1 \leq j \leq n_{23}, \\ e_{i j}^{k} e_{\ell m}^{h}=0 & \text { for } j \neq \ell .\end{cases}
$$

LEMMA 4.1. With respect to the multiplication (4.5) the euclidean vector space $N$ is an $N$-algebra of type I having $S$ as its diagram. Every $N$-algebra of type $I$ of rank 3 having $S$ as its diagram can be obtained in this way, provided that the value of the parameter $t$ is suitably chosen.

Proof. The multiplication (4.5) satisfies (N1) and (N2). The associativity and (N5) are also trivially satisfied. Let $\langle$,$\rangle be the inner product$ of $N$. Using (4.4) and (4.5) we have

$$
\begin{aligned}
& \left\langle e_{12}^{i} e_{23}^{j}, e_{12}^{k} e_{23}^{\ell}\right\rangle+\left\langle e_{12}^{i} e_{23}^{\ell}, e_{12}^{k} e_{23}^{j}\right\rangle \\
& \quad=\sum_{s} b_{i j}^{s} b_{k \ell}^{s}+\sum_{s} b_{i \ell}^{s} b_{k j}^{s}=a_{(i j)(k \ell)}+a_{(i \ell)(k j)} \\
& =\frac{2}{n_{2}} \delta_{i k} \delta_{j \ell}
\end{aligned}
$$

which proves $\left(\mathrm{N}^{\prime \prime}\right)$. The first assertion of the lemma was thus proved. Let $N=N_{12}+N_{23}+N_{13}$ be an $N$-algebra of type I of rank 3 with $S$ as its diagram, and let $\left\{e_{i j}^{k}\right\}$ be an orthonormal base of $N_{i j}$. We define the matrix $A=\left(a_{(i j)(k \ell)}\right)$ by putting $a_{(i j)(k \ell)}=\left\langle e_{12}^{i} e_{23}^{j}, e_{12}^{k} e_{23}^{\ell}\right\rangle$. Then $A$ satisfies (4.1)-(4.3) and coincides with the Grammian $A_{t}$ of $S$ for a fixed value of the parameter $t$. Since $e_{12}^{i} e_{23}^{j}$ is written in the form $\sum_{k=1}^{n_{13}} c_{i j}^{k} e_{13}^{k}$, we have $a_{(i j)(k \ell)}=\sum_{s} c_{i j}^{s} c_{k \ell}^{s}$, which implies $A=B_{0}^{t} B_{0}$, where $B_{0}=\left(c_{i j}^{k}\right)$. This means that the matrix $B_{0}$ of the structure constants of $N$ is a solution of (4.4).

q.e.d.

For an $N$-algebra $N$ having $S$ as its diagram, the matrix $A=$ $\left(\left\langle e_{12}^{i} e_{23}^{j}, e_{12}^{k} e_{23}^{\ell}\right\rangle\right)$ is called the Grammian of $N$ with respect to the orthonormal bases $\left\{e_{i j}^{k}\right\}$. In what follows, an $N$-algebra $N$ having $S$ as its diagram is often called an $N$-algebra corresponding to $S$.

Lemma 4.2. Let $N=N_{12}+N_{23}+N_{13}$ and $N^{\prime}=N_{12}^{\prime}+N_{23}^{\prime}+N_{13}^{\prime}$ be two $N$-algebras of type I corresponding to $S$. Let $\left\{e_{i j}^{k}\right\}$ (resp. $\left.\left\{e_{i j}^{\prime k}\right\}\right)$ be an orthonormal base of $N_{i j}$ (resp. $\left.N_{i j}^{\prime}\right)(1 \leq i<j \leq 3)$, and let $B=\left(b_{i j}^{k}\right)$ 
(resp. $B^{\prime}=\left(b_{i j}^{\prime k}\right)$ ) be the matrix of the structure constants of $N$ (resp. $N^{\prime}$ ) with respect to these bases. Then $N$ is isomorphic to $N^{\prime}$ if and only if there exist matrices $T_{1} \in O\left(n_{12}\right), T_{2} \in O\left(n_{23}\right)$ and $T_{3} \in O\left(n_{13}\right)$ such that

$$
\left(T_{1} \otimes T_{2}\right) B=B^{\prime} T_{3} .
$$

Proof. Suppose that there exist such matrices $T_{1}, T_{2}$ and $T_{3}$. Put $T_{1}=\left(\alpha_{k i}\right), T_{2}=\left(\beta_{\ell j}\right)$ and $T_{3}=\left(\gamma_{t s}\right)$. By (4.6) we have

$$
\sum_{s} b_{i j}^{s} \gamma_{t s}=\sum_{\ell, k} \alpha_{k i} \beta_{\ell j} b_{k \ell}^{\prime t}
$$

We define the linear isometry $\varphi$ of $N$ onto $N^{\prime}$ by $\varphi \mid N_{i j}=\varphi_{i j}$, where $\varphi_{12}\left(e_{12}^{i}\right)=\sum_{k} \alpha_{k i} e_{12}^{\prime k}, \varphi_{23}\left(e_{23}^{j}\right)=\sum_{\ell} \beta_{\ell j} e_{23}^{\prime \ell}$ and $\varphi_{13}\left(e_{13}^{k}\right)=\sum_{s} \gamma_{s k} e_{13}^{\prime s}$. Then $\varphi$ is an isomorphism of $N$ onto $N^{\prime}$; in fact

$$
\begin{aligned}
\varphi\left(e_{12}^{i}\right) \varphi\left(e_{23}^{j}\right) & =\sum_{k, \ell} \alpha_{k i} \beta_{\ell j} e_{12}^{\prime t} e_{23}^{\prime \ell}=\sum_{k, \ell, t} \alpha_{k i} \beta_{\ell j} b_{k l}^{t} e_{13}^{\prime t} \\
& =\sum_{s, t} b_{i j}^{s} \gamma_{t s} e_{13}^{\prime t}=\varphi\left(e_{12}^{i} e_{23}^{j}\right) .
\end{aligned}
$$

The "only if" part is analogously proved.

q.e.d.

LEMMA 4.3. Let $B_{1}$ and $B_{2}$ be $n \times m$ real matrices such that $B_{1}^{t} B_{1}$ $=B_{2}^{t} B_{2}$. Then there exists a matrix $T_{2} \in O(m)$ such that $B_{2}=B_{1} T_{2}$.

Proof. Let us put $A=B_{1}^{t} B_{1}$. Let $\left\{\alpha_{1}^{2}, \cdots, \alpha_{s}^{2}\right\}$ be the set of all nonzero different eigenvalues of $A$. We assume that $\alpha_{1}>\alpha_{2}>\cdots>\alpha_{s}>0$. There exists a matrix $U \in O(n)$ such that $A=U D^{t} U$, where

$$
D=\left(\begin{array}{cccc}
\alpha_{1}^{2} E_{n_{1}} & & & \\
& \ddots & & \\
& & \alpha_{s}^{2} E_{n_{s}} & \\
& & & 0
\end{array}\right)
$$

Noting that $\operatorname{rank} A \leq n, m$, we define the $n \times m$ real matrix $D_{0}$ as

$$
D_{0}=\left(\begin{array}{cccc}
\alpha_{1} E_{n_{1}} & & & \\
& \ddots & & \\
& & \alpha_{s} E_{n_{s}} & \\
& & & 0
\end{array}\right) .
$$

and put $B_{0}=U D_{0}$. Then $A=B_{0}^{t} B_{0}$ holds*). So, in proving the lemma,

*) This method of finding $B_{0}$ will be used in the proofs of the propositions $5.3,6.3$, $6.4,6.5,6.6$ in order to find a solution $B$ of (4.4). A simpler proof of Lemma 4.3 was kindly informed us by the referee. 
without loss of generality we can assume that $B_{1}=B_{0}$. Since $B_{0}^{t} B_{0}=$ $B_{2}^{t} B_{2}$, there exist the matrices $T \in O(n)$ and $T^{\prime} \in O(m)$ such that $B_{2}=T B_{0} T^{\prime}$, as is known in the matrix theory. We have $T A=A T$; in fact $T A^{t} T=$ $T\left(B_{0}^{t} B_{0}\right)^{t} T=T B_{0}^{t}\left(T B_{0}\right)=\left(B_{2}^{t} T^{\prime}\right)^{t}\left(B_{2}^{t} T^{\prime}\right)=A$. So, putting $Y={ }^{t} U T U$, we have $Y D=D Y$ (cf. [2]). Since $D$ is the diagonal matrix given by (4.7) and commutes with $Y$, it follows from the direct verification that $Y$ is written in the form

$$
Y=\left(\begin{array}{lllll}
X_{1} & & & & \\
& X_{2} & & & \\
& & \ddots & & \\
& & & X_{s} & \\
& & & X_{s+1}
\end{array}\right),
$$

where $X_{i}(1 \leq i \leq s)$ is a matrix of degree $n_{i}$. By the definition of $Y$ each $X_{i}$ is an orthogonal matrix. We define the orthogonal matrix $T_{1}^{\prime}$ of degree $m$ by

$$
T_{1}^{\prime}=\left(\begin{array}{lllll}
X_{1} & & & & \\
& X_{2} & & & \\
& & \ddots & & \\
& & & X_{s} & \\
& & & E_{k}
\end{array}\right) \text {, }
$$

where $k=m-\left(n_{1}+n_{2}+\cdots+n_{s}\right)$. Then an easy computation shows that $Y D_{0}=D_{0} T_{1}^{\prime}$. Therefore $B_{2}=T B_{0} T^{\prime}=\left(U Y^{t} U\right)\left(U D_{0}\right) T^{\prime}=U Y D_{0} T^{\prime}=$ $U D_{0} T_{1}^{\prime} T^{\prime}=B_{0} T_{2}$, where $T_{2}=T_{1}^{\prime} T^{\prime}$.

q.e.d.

CoRollary 4.4. Let $N$ and $N^{\prime}$ be two $N$-algebras of type I corresponding to the skeleton $S$. Let $A$ (resp. $A^{\prime}$ ) be the Grammian of $N$ (resp. $N^{\prime}$ ) for some fixed orthonormal bases. If $A=A^{\prime}$, then $N$ is isomorphic to $N^{\prime}$.

Proof. Let $B$ (resp. $B^{\prime}$ ) be the matrix of the structure constants of $N$ (resp. $N^{\prime}$ ) with respect to the given bases. Then, by the assumption, we have $B^{t} B=B^{\prime t} B^{\prime}$. By Lemma 4.3 there exists an orthogonal matrix $T_{3}$ such that $B^{\prime}=B T_{3}$. Hence the corollary is immediate from Lemma 4.2.

Proposition 4.5. Let $A_{t}$ be the Grammian of the skeleton $S$ with $n_{12}=2$ or $n_{23}=2$. Let $N_{1}$ and $N_{2}$ be the $N$-algebras of type $I$, having 
$S$ as their diagrams, which correspond to fixed values $s_{1}$ and $s_{2}$ of the parameter $t$, respectively. Then $N_{1}$ and $N_{2}$ are isomorphic if and only if the Grammians $A_{s_{1}}$ and $A_{s_{2}}$ have the same eigenvalues.

Proof. Suppose that $N_{1}$ is isomorphic to $N_{2}$. Then the assertion is an immediate consequence of (4.6). To prove the converse, let us first consider the case of $n_{12}=2$. Then, taking (4.1) and (4.2) into account, we can see that the Grammian $A_{t}$ is written as

$$
\left(\begin{array}{cc}
\frac{1}{n_{2}} E_{n_{23}} & -\tilde{A}_{t} \\
\tilde{A}_{t} & \frac{1}{n_{2}} E_{n_{23}}
\end{array}\right) \text {, }
$$

where $\tilde{A}_{t}$ is a skew-symmetric matrix depending on the parameter $t$. Hence we can write $A_{t}$ in the form

$$
A_{t}=\left(\begin{array}{rr}
0 & -1 \\
1 & 0
\end{array}\right) \otimes \tilde{A}_{t}+\frac{1}{n_{2}} E_{2 n_{23}}
$$

for each $t$. Consequently, from the assumption of the proposition it follows that the skew-symmetric matrices $\tilde{A}_{s_{1}}$ and $\tilde{A}_{s_{2}}$ have the same eigenvalues. So there exists a matrix $T \in O\left(n_{23}\right)$ such that $T \tilde{A}_{s_{1}}{ }^{t} T=\tilde{A}_{s_{2}}$. Let $B_{s_{i}}(i=$ $1,2)$ be the matrix of the structure constants of $N_{i}$ with respect to some orthonormal bases. Then $B_{s_{i}}$ is a solution of the equation (4.4) for $t=$ $s_{i}(i=1,2)$. Putting $B^{\prime}=\left(E_{2} \otimes T\right) B_{s_{1}}$, we have $B^{\prime t} B^{\prime}=A_{s_{2}}$. Hence, by Lemma 4.3, there exists an orthogonal matrix $T^{\prime}$ such that $B^{\prime}=B_{s_{2}} T^{\prime}$. We have thus $\left(E_{2} \otimes T\right) B_{s_{1}}=B_{s_{2}} T^{\prime}$, which implies that $N_{1}$ and $N_{2}$ are isomorphic (cf. Lemma 4.2).

Next, let us consider the case of $n_{23}=2$. Then, taking (4.1) and (4.2) into account, the Grammian $A_{t}$ is seen to be

$$
A_{t}=\tilde{A}_{t} \otimes\left(\begin{array}{rr}
0 & -1 \\
1 & 0
\end{array}\right)+\frac{1}{n_{2}} E_{2 n_{12}},
$$

where $\tilde{A}_{t}$ is a skew-symmetric matrix depending on the parameter $t$. Hence, by the same way as in the case of $n_{12}=2$, we can conclude that $N_{1}$ is isomorphic to $N_{2}$. q.e.d.

4.2. Let us consider the following two skeletons 

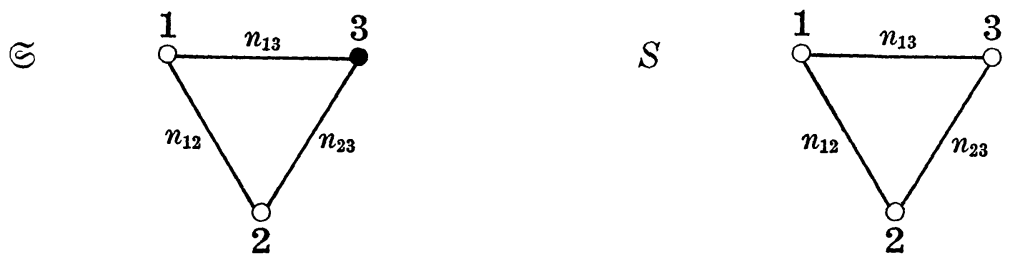

and let $N=N_{12}+N_{23}+N_{13}$ be an $N$-algebra of type I corresponding to $S$; let $B=\left(b_{i j}^{k}\right)$ be the matrix of the structure constants of $N$ relative to orthonormal bases $\left\{e_{i j}^{k}\right\}$ of $N_{i j}$. Then we have

LEMMA 4.6. The N-algebra $N$ of type $I$ is that of type II corresponding to the skeleton $\mathbb{S}$ if and only if there exist matrices $J_{13} \in O\left(n_{13}\right)$ and $J_{23} \in O\left(n_{23}\right)$ such that

$$
\left\{\begin{array}{c}
J_{13}^{2}=-E_{n_{13}}, \quad J_{23}^{2}=-E_{n_{23}}, \\
\left(E_{n_{12}} \otimes J_{23}\right) B=B J_{13} ;
\end{array}\right.
$$

in this case the complex structure of $N$ is given by the pair $\left(J_{13}, J_{23}\right)$.

Proof. Suppose that there exist such matrices $J_{13}$ and $J_{23}$. Put $J_{13}$ $=\left(\alpha_{t s}\right), J_{23}=\left(\beta_{\ell j}\right)$. Then, from (4.8) we have

$$
\sum_{s} b_{i j}^{s} \alpha_{t s}=\sum_{k, \ell} \delta_{k i} \beta_{\ell j} b_{k \ell}^{t}=\sum_{\ell} \beta_{\ell j} b_{i \ell}^{t} .
$$

Let $j_{i 3}(i=1,2)$ be the orthogonal transformation defined by $J_{i 3}$ with respect to the bases $\left\{e_{i j}^{k}\right\}$. Then the above equality implies $j_{13}\left(e_{12}^{i} e_{23}^{j}\right)=$ $e_{12}^{i}\left(j_{23} e_{23}^{j}\right)$. And the pair $\left(j_{13}, j_{23}\right)$ is the desired complex structure on $N$ (cf. Definition 2.3). The converse is immediate.

q.e.d.

In view of the above lemma we can regard the complex structure of the $N$-algebra $N$ of type II as the pair $\left(J_{13}, J_{23}\right)$ of the orthogonal matrices satisfying (4.8).

DEFinition 4.7. Let $N$ be an $N$-algebra of type I corresponding to the skeleton $S$, and let $B$ be the matrix of the structure constants with respect to orthonormal bases. Let $J=\left(J_{13}, J_{23}\right)$ and $J^{\prime}=\left(J_{13}^{\prime}, J_{23}^{\prime}\right)$ be two complex structures on $N$. Then $J$ is said to be equivalent to $J^{\prime}$ (or, simply denoted by $\left.J \sim J^{\prime}\right)$ if there exist three matrices $T_{1} \in O\left(n_{12}\right), T_{2} \in O\left(n_{23}\right)$ and $T_{3} \in O\left(n_{13}\right)$ such that

$$
\begin{aligned}
\left(T_{1} \otimes T_{2}\right) B & =B T_{3}, \\
T_{2} J_{23} & =J_{23}^{\prime} T_{2},
\end{aligned}
$$

and 


$$
T_{3} J_{13}=J_{13}^{\prime} T_{3}
$$

This is obviously an equivalence relation. From Lemma 4.2 and Definition 2.5 we have immediately

LEMMA 4.8. Let $(N, J)$ and $\left(N, J^{\prime}\right)$ be two $N$-algebra structures of type II on the N-algebra $N$ of type I in Definition 4.7. Then $(N, J)$ is isomorphic to $\left(N, J^{\prime}\right)$ if and only if $J$ is equivalent to $J^{\prime}$.

LEMMA 4.9. Let $\left(S,\left(n_{i j}\right)\right)$ (resp. $\left(\widetilde{S},\left(m_{i j}\right)\right)$ ) be an m-skeleton of type $I$ (resp. type II) satisfying either the condition $(P)$ or $\left(P^{\prime}\right)$;

(P) for each triple $(i, j, k)$ of vertices such that $i<j<k$, the condition $i \neq j$ or $j \neq k$ is valid.

$\left(P^{\prime}\right) \quad m \leq 2$ for $\left(S,\left(n_{i j}\right)\right)$ (resp. $m=1$ for $\left(\widetilde{S},\left(m_{i j}\right)\right)$ ).

Then there exists a unique N-algebra of type $I$ (resp. type II) corresponding to $\left(S,\left(n_{i j}\right)\right)$ (resp. $\left.\left(S_{,},\left(m_{i j}\right)\right)\right)$; in this case the product of any two elements is always zero.

Proof. We will prove the lemma only for the case that $\left(\widetilde{S},\left(m_{i j}\right)\right)$ satisfies $(P)$, since other cases are similar. Let $N=\sum_{1 \leq i<j \leq m+1} N_{i j}$ be an euclidean vector space such that the right-hand side is the orthogonal direct sum of $N_{i j}$ 's, where $\operatorname{dim} N_{i j}=m_{i j}$. Since $N$ must satisfy (N4) and (N2), it follows from $(P)$ that the product of two elements of $N$ should be zero; with this multiplication, $N$ is an $N$-algebra of type I. Since $m_{i, m+1}$ is even, we can find a complex structure $j_{i, m+1}$ on $N_{i, m+1}$ $(1 \leq i \leq m)$ which leaves the given inner product invariant. $N$ is thus an $N$-algebra of type II corresponding to $\left(\mathfrak{S},\left(m_{i j}\right)\right)$. If we change the inner product and the complex structure to another, then the $N$-algebra structure of type II remains isomorphic, since two hermitian vector spaces of the same dimension are isomorphic. q.e.d.

Remark 4.10. Suppose that there exists a unique $N$-algebra $N$ corresponding to a given skeleton $S$ of type I or type II. Then every $N$ algebra whose diagram is isomorphic to $S$ is isomorphic to $N$.

\section{\$ 5. Classification of $N$-algebras of type $I$}

Throughout this section we will call, for brevity, an $N$-algebra of type I an $N$-algebra. Let $\left\{e_{i j}^{k}\right\}$ always denote an orthonormal base of the euclidean space $N_{i j}$ and $\langle$,$\rangle denote the inner product of an N$-algebra. As a corollary to Lemma 4.9 we have 
Proposition 5.1. There exists a unique $N$-algebra $N$ whose diagram is one of the skeletons $S_{1}, S_{2}, S_{3}^{1}, S_{3}^{1^{*}}, S_{4}^{1}, S_{4}^{1^{*}}, S_{4}^{2}, S_{4}^{3}, S_{5}^{1}, S_{5}^{1^{*}}, S_{5}^{2}, S_{5}^{2^{*}}, S_{5}^{3}, S_{5}^{3^{*}}, S_{5}^{4}, S_{5}^{4^{*}}$ in Proposition 3.5; the product of any two elements is zero.

Proposition 5.2. There exists a unique N-algebra whose diagram is $S_{3}^{2}$ with $n_{12}=1$ or $n_{23}=1$; the multiplications are as follows;

$$
\begin{array}{ll}
e_{12}^{1} e_{23}^{i}=\sqrt{\frac{2}{n_{23}+3}} e_{13}^{i} \quad\left(1 \leq i \leq n_{23}\right) \quad \text { for } n_{12}=1 \\
e_{12}^{i} e_{23}^{1}=\sqrt{\frac{2}{n_{12}+3} e_{13}^{i}} \quad\left(1 \leq i \leq n_{12}\right) \quad \text { for } n_{23}=1
\end{array}
$$

Proof. We consider only the case of $n_{12}=1$. The Grammian $A_{t}$ of the skeleton is given by $\left(2 /\left(n_{23}+3\right)\right) E_{n_{23}}$. The $n_{23} \times n_{13}$ matrix $B=$ $\sqrt{2 /\left(n_{23}+3\right)}\left(E_{n_{23}}, 0\right)$ is a solution of the equation (4.4), from which we get the multiplication (5.1). Since the Grammian $A_{t}$ is a constant matrix, the uniqueness follows from Corollary 4.4 and Lemma 4.1. q.e.d.

Proposition 5.3. There exists a unique N-algebra whose diagram is $S_{3}^{2}$ with $n_{12}=n_{23}=2$ and $n_{13}=3$; the multiplication is as follows;

$$
\begin{cases}e_{12}^{1} e_{23}^{1}=\frac{1}{\sqrt{3}} e_{13}^{1}, & e_{12}^{2} e_{23}^{1}=\frac{1}{\sqrt{3}} e_{13}^{2}, \\ e_{12}^{1} e_{23}^{2}=\frac{1}{\sqrt{3}} e_{13}^{2}, & e_{12}^{2} e_{23}^{2}=-\frac{1}{\sqrt{3}} e_{13}^{1} .\end{cases}
$$

Proof. Putting $a_{(12)(21)}=t$, the Grammian $A_{t}$ of the given skeleton is

$$
A_{t}=\left(\begin{array}{rr}
0 & -1 \\
1 & 0
\end{array}\right) \otimes\left(\begin{array}{rr}
0 & t \\
-t & 0
\end{array}\right)+\frac{1}{3} E_{4} \text {. }
$$

The eigenvalues of $A_{t}$ are $\frac{1}{3} \pm t$ both with multiplicity two. As we remarked in $\S 4$, the equation (4.4) has a solution if and only if $\operatorname{rank} A_{t} \leq$ $n_{13}=3$, from which we get $t= \pm \frac{1}{3}$. We have thus two Grammians $A_{1 / 3}$ and $A_{-1 / 3}$. Since $A_{1 / 3}$ and $A_{-1 / 3}$ have the same eigenvalues, the corresponding two $N$-algebra structures are isomorphic (cf. Proposition 4.5). The uniqueness in the proposition follows from this and Lemma 4.1. Put

$$
B_{1 / 3}=\frac{1}{\sqrt{3}}\left(\begin{array}{rrr}
1 & 0 & 0 \\
0 & 1 & 0 \\
0 & 1 & 0 \\
-1 & 0 & 0
\end{array}\right)
$$


Then $B_{1 / 3}$ is a solution of the equation $A_{1 / 3}=B^{t} B$, from which we get (5.2). q.e.d.

A result of Vinberg [9] shows that there exists a unique $N$-algebra corresponding to $S_{3}^{2}$ with $n_{12}=n_{23}=n_{13}=2$ and that the multiplication is given by (5.2).

Proposition 5.4. There exists a unique N-algebra whose diagram is one of the skeletons $S_{4}^{4}, S_{4}^{4^{*}}, S_{5}^{5}, S_{5}^{5^{*}}, S_{5}^{6}, S_{5}^{6^{*}}$ and $S_{5}^{7}$ in Proposition 3.5.

Proof. Note that for $S_{4}^{4} n_{12}$ or $n_{24}$ is equal to one and that for $S_{4}^{4^{*}} n_{13}$ or $n_{34}$ is equal to one. The proposition is easily seen from the proof of Proposition 5.2 and Lemma 4.9.

Letting $a$ be an element of an $N$-algebra $N$, we denote by $L_{a}\left(\operatorname{resp} . R_{a}\right)$ the left (resp. right) multiplication by $a$ in the $N$-algebra $N$.

Proposition 5.5. There exists a unique N-algebra corresponding to the skeleton $S_{4}^{5}$ (resp. $S_{4}^{\left.5^{*}\right)}$; the multiplication is as follows;

$$
\begin{array}{r}
\left\{\begin{array}{l}
e_{12}^{1} e_{23}^{1}=\sqrt{\frac{2}{5}} e_{13}^{1}, \\
e_{12}^{1} e_{24}^{1}=\sqrt{\frac{2}{5}} e_{14}^{1},
\end{array}\right. \\
\text { (resp. }\left\{\begin{array}{l}
e_{13}^{1} e_{34}^{1}=\sqrt{\frac{2}{5}} e_{14}^{1} \\
e_{23}^{1} e_{34}^{1}=\sqrt{\frac{2}{5}} e_{24}^{1}
\end{array}\right.
\end{array}
$$

Proof. We give the proof only for the case of $S_{4}^{5}$. Let $N=N_{12}+$ $N_{23}+N_{13}+N_{24}+N_{14}$ be the orthogonal direct sum of the euclidean vector spaces $N_{i j}$ of dimension $n_{i j}$, where $n_{12}=n_{23}=n_{13}=n_{24}=1$ and $n_{14}=1$ or 2. Suppose that $N$ has the algebra structure whose multiplication is given by (5.4) and (N2). Then it is easy to see that $N$ is an $N$-algebra having $S_{4}^{5}$ as its diagram.

Let $N^{\prime}=\sum N_{i j}^{\prime}$ be another $N$-algebra corresponding to $S_{4}^{5}$. The subspaces $M=N_{12}+N_{24}+N_{14}$ and $M^{\prime}=N_{12}^{\prime}+N_{24}^{\prime}+N_{14}^{\prime}$ are subalgebras of $N$ and $N^{\prime}$, respectively, whose multiplications satisfy (N4). Hence, as is seen from the proof of Proposition 5.2 there exists an algebra isomorphism $\varphi_{1}$ of $M$ onto $M^{\prime}$, which is also isometric and grade-preserving. Let $\varphi_{2}$ be a natural isometry of the vector space $N_{23}$ onto $N_{23}^{\prime}$. The right 
multiplication $R_{e_{23}^{1}}$ is a bijection of $N_{12}$ onto $N_{13}$ by virtue of (N4). Let $\varphi$ be a linear isomorphism of $N$ onto $N^{\prime}$ defined by

$$
\varphi= \begin{cases}\varphi_{1} & \text { on } M, \\ \varphi_{2} & \text { on } N_{23}, \\ R_{\varphi_{2}\left(e_{23}^{1}\right)} \circ \varphi_{1} \circ R_{e_{23}^{1}}^{-1} & \text { on } N_{13} .\end{cases}
$$

Then $\varphi$ is an isometry and isomorphism; in fact, $\varphi\left(e_{12}^{1} e_{23}^{1}\right)=R_{\varphi_{2}\left(e_{23}^{1}\right)} \circ \varphi_{1}$ $\circ R_{e_{23}^{1}}^{-1}\left(e_{12}^{1} e_{23}^{1}\right)=\varphi_{1}\left(e_{12}^{1}\right) \varphi_{2}\left(e_{23}^{1}\right)=\varphi\left(e_{12}^{1}\right) \varphi\left(e_{23}^{1}\right)$.

Proposition 5.6. There exists a unique N-algebra corresponding to the skeleton $S_{4}^{6}$; the multiplication is given by

$$
\left\{\begin{array}{l}
e_{12}^{1} e_{24}^{1}=\frac{1}{\sqrt{2}} e_{14}^{1} \\
e_{13}^{1} e_{34}^{1}=\frac{1}{\sqrt{2}} e_{14}^{2} .
\end{array}\right.
$$

Proof. Let $N=N_{12}+N_{24}+N_{14}+N_{13}+N_{34}$ be the orthogonal direct sum of the euclidean vector spaces $N_{i j}$ of dimension $n_{i j}$, where $n_{12}=n_{24}$ $=n_{13}=n_{34}=1$ and $n_{14}=2$. We can easily see that if the multiplication (5.5) and (N2) is given to $N$, then $N$ is an $N$-algebra corresponding to $S_{4}^{6}$.

Let $N^{\prime}=\sum N_{i j}^{\prime}$ be another $N$-algebra corresponding to $S_{4}^{6}$. Put $M_{1}$ $=N_{13}+N_{34}+N_{13} N_{34}, \quad M_{2}=N_{12}+N_{24}+N_{12} N_{24}, \quad M_{1}^{\prime}=N_{13}^{\prime}+N_{34}^{\prime}+N_{13}^{\prime} N_{34}^{\prime}$ and $M_{2}^{\prime}=N_{12}^{\prime}+N_{24}^{\prime}+N_{12}^{\prime} N_{24}^{\prime}$. Then $M_{1}$ and $M_{2}$ (resp. $M_{1}^{\prime}$ and $M_{2}^{\prime}$ ) are ideals of $N$ (resp. $N^{\prime}$ ). By (N5) we have $\left\langle N_{13} N_{34}, N_{12} N_{24}\right\rangle=\left\langle N_{13}^{\prime} N_{34}^{\prime}, N_{12}^{\prime} N_{24}^{\prime}\right\rangle$ $=0$. So $N$ (resp. $N^{\prime}$ ) is the direct sum of ideals $M_{1}$ and $M_{2}$ (resp. $M_{1}^{\prime}$ and $M_{2}^{\prime}$ ). On the other hand, from the proof of Proposition 5.2, it follows that there exists an algebra isomorphism $\varphi_{i}$ of $M_{i}$ onto $M_{i}^{\prime}(i=$ $1,2)$ which is also isometric and grade-preserving. The map $\varphi$ of $N$ onto $N^{\prime}$ defined by $\varphi \mid M_{i}=\varphi_{i}(i=1,2)$ is an isomorphism of $N$ onto $N^{\prime}$.

q.e.d.

According to Vinberg [9] there exists a unique $N$-algebra corresponding to $S_{4}^{7}$; the multiplication is given by

$$
\begin{cases}e_{12}^{1} e_{23}^{1}=\sqrt{\frac{2}{5}} e_{13}^{1}, & e_{13}^{1} e_{34}^{1}=\sqrt{\frac{2}{5}} e_{14}^{1}, \\ e_{12}^{1} e_{24}^{1}=\sqrt{\frac{2}{5}} e_{14}^{1}, & e_{23}^{1} e_{34}^{1}=\sqrt{\frac{2}{5}} e_{24}^{1} .\end{cases}
$$

In view of the results in this section and Remark 4.10 we have 
worked out the classification of $N$-algebras of type I of the problem III) in $\S 3$.

\section{§ 6. Classification of $\boldsymbol{N}$-algebras of type II}

Throughout this section, for simplicity, we will call an $\mathrm{N}$-algebra of type II an $N$-algebra. $I(\varepsilon)$ denotes the matrix $\left(\begin{array}{rr}0 & -\varepsilon \\ \varepsilon & 0\end{array}\right)$ for $\varepsilon= \pm 1$. Let $A$ and $B$ be arbitrary two matrices. Then we define the direct sum $A \oplus B$ by $\left(\begin{array}{cc}A & 0 \\ 0 & B\end{array}\right)$. In following each proposition, complex structures and multiplications are represented with respect to the same orthonormal bases $\left\{e_{i j}^{k}\right\}$.

As a corollary to Lemma 4.9 we get

Proposition 6.1. There exists a unique $N$-algebra whose diagram is one of the skeletons $\mathfrak{S}_{1}, \mathfrak{S}_{2}^{1}, \mathfrak{S}_{3}^{1}, \mathfrak{S}_{3}^{3}, \mathfrak{S}_{3}^{4}, \mathfrak{S}_{4}^{1}, \mathfrak{S}_{4}^{2}, \mathfrak{S}_{4}^{3}, \mathfrak{S}_{4}^{4}$ in Proposition 3.6.

Proposition 6.2. There exists a unique N-algebra whose diagram is $\mathfrak{S}_{2}^{2}$ with $n_{12}=1$. Furthermore the multiplication is given by (5.1) and the complex structure is represented as follows;

$$
J_{i 3}=I(1) \oplus \cdots \oplus I(1) \quad\left(\frac{n_{i 3}}{2}-\text { copies }\right), \quad i=1,2 .
$$

Proof. By Proposition 5.2, there exists a unique $N$-algebra $N$ of type I whose diagram is $S$ with $n_{12}=1$ in $\S 4$ and the matrix $B$ of the structure constants is given by $B=\sqrt{\left(2 /\left(n_{23}+3\right)\right)}\left(E_{n_{23}} 0\right)$. Let $J_{13} \in O\left(n_{13}\right)$ and $J_{23} \in O\left(n_{23}\right)$ and let us decompose $J_{13}$ into submatrices as follows;

$$
J_{13}=\left(\begin{array}{ll}
J_{13}^{(1)} & J_{13}^{(3)} \\
J_{13}^{(4)} & J_{13}^{(2)}
\end{array}\right),
$$

where $J_{13}^{(1)}$ (resp. $J_{13}^{(2)}$ ) is a square matrix of degree $n_{23}$ (resp. $n_{13}-n_{23}$ ). Then it is verified that $J=\left(J_{13}, J_{23}\right)$ satisfies (4.8) if and only if $J_{13}^{(1)}=$ $J_{23}, J_{13}^{(3)}=0, J_{13}^{(4)}=0, J_{13}^{(1) 2}=-E_{n_{23}}, J_{13}^{(2) 2}=-E_{n_{13}-n_{23}}$. We define $\tilde{J}=\left(\tilde{J}_{13}\right.$, $\tilde{J}_{23}$ ) satisfying (4.8) by

$$
\tilde{J}_{i 3}=I(1) \oplus \cdots \oplus I(1) \quad\left(\frac{n_{i 3}}{2}-\text { copies }\right), \quad i=1,2 .
$$

It can be seen that there exist two matrices $T_{1} \in O\left(n_{23}\right)$ and $T_{2} \in O\left(n_{13}-n_{23}\right)$ such that 


$$
T_{1} J_{23}=\tilde{J}_{23} T_{1}, \quad\left(T_{1} \oplus T_{2}\right) J_{13}=\tilde{J}_{13}\left(T_{1} \oplus T_{2}\right)
$$

Since $T_{1} B=B\left(T_{1} \oplus T_{2}\right)$, it follows from Lemma 4.8 that $(N, J)$ is isomorphic to $(N, \tilde{J})$.

q.e.d.

Proposition 6.3. There exists a unique N-algebra whose diagram is $\widetilde{\varsigma}_{2}^{2}$ with $\left(n_{12}, n_{23}, n_{13}\right)=(2,2,2)$. The multiplication is given by (5.2) and the complex structure is represented by $J_{13}=J_{23}=I(1)$.

Proof. As we remarked before Proposition 5.4, there exists a unique $N$-algebra $N$ of type I whose diagram is $S_{3}^{2}$ with $\left(n_{12}, n_{23}, n_{13}\right)=(2,2,2)$ in Proposition 3.5 and the matrix $B$ of the structure constants is given by

$$
B=\frac{1}{\sqrt{3}}\left(\begin{array}{rr}
1 & 0 \\
0 & 1 \\
0 & 1 \\
-1 & 0
\end{array}\right)
$$

Let $J=\left(J_{13}, J_{23}\right)$ be a pair of the orthogonal matrices of degree 2 . Then $J$ satisfies (4.8) if and only if $J_{13}=J_{23}=I(\varepsilon)$. Put $J^{(\varepsilon)}=\left(J_{13}^{(\varepsilon)}, J_{23}^{(\varepsilon)}\right)$, where $J_{13}^{(\varsigma)}=J_{23}^{(\varsigma)}=I(\varepsilon)$. Then $J^{(1)}$ is equivalent to $J^{(-1)}$. In fact, $T=\left(\begin{array}{rr}1 & 0 \\ 0 & -1\end{array}\right)$ satisfies the equalities $T J_{i 3}^{(1)}=J_{i 3}^{(-1)} T(i=1,2)$ and $(T \otimes T) B=B T$.

q.e.d.

Proposition 6.4. The N-algebras whose diagrams are $\mathfrak{S}_{2}^{2}$ with $\left(n_{12}\right.$, $\left.n_{23}, n_{13}\right)=(2,2,4)$ or $(2,2,6)$ are effectively parametrized by the closed interval $\left[0, \frac{1}{3}\right]$; the multiplication and the complex structure of the $\mathrm{N}$ algebra $N_{t}$ corresponding to $t \in\left[0, \frac{1}{3}\right]$ are given as follows;

$$
\begin{array}{ll}
e_{12}^{1} e_{23}^{1}=\lambda e_{13}^{1}+\mu e_{13}^{3}, & e_{12}^{2} e_{23}^{1}=\lambda e_{13}^{2}-\mu e_{13}^{4}, \\
e_{12}^{1} e_{23}^{2}=\lambda e_{13}^{2}+\mu e_{13}^{4}, & e_{12}^{2} e_{23}^{2}=-\lambda e_{13}^{1}+\mu e_{13}^{3},
\end{array}
$$

where $\lambda=\sqrt{(1+3 t) / 6}, \mu=\sqrt{(1-3 t) / 6}$.

$$
J_{13}=\left\{\begin{array}{ll}
I(1) \oplus I(1) & \text { for } n_{13}=4 \\
I(1) \oplus I(1) \oplus I(1) & \text { for } n_{13}=6
\end{array}, J_{23}=I(1)\right.
$$

Proof. First we show that $N$-algebras of type I whose diagrams are $S$ in $\S 4$ satisfying $\left(n_{12}, n_{23}, n_{13}\right)=(2,2,4)$ or $(2,2,6)$ are effectively parametrized by $\left[0, \frac{1}{3} .^{*}\right.$ ) The Grammians $A_{t}$ of these two skeletons are the

*) The existence of a one-parameter family of non-isomorphic $N$-algebras of type I corresponding to $S$ with $\left(n_{12}, n_{23}, n_{13}\right)=(2,2,4)$ has been stated in Vinberg [8]. 
same as in (5.3) and the eigenvalues of $A_{t}$ are $\frac{1}{3} \pm t$ both with multiplicity 2 (cf. Proposition 5.3). Since $A_{t}$ is positive semi-definite, it follows that $-\frac{1}{3} \leq t \leq \frac{1}{3}$. Since $\operatorname{rank} A_{t} \leq n_{13}=4$ or 6 , the solutions of the equation (4.4) always exist and one of the solutions is given by

$$
\begin{aligned}
& B_{t}=\left[\begin{array}{rrrr}
\lambda & 0 & \mu & 0 \\
0 & \lambda & 0 & \mu \\
0 & \lambda & 0 & -\mu \\
-\lambda & 0 & \mu & 0
\end{array}\right) \quad \text { for } n_{13}=4, \\
& B_{t}=\left(\begin{array}{rrrrrr}
\lambda & 0 & \mu & 0 & 0 & 0 \\
0 & \lambda & 0 & \mu & 0 & 0 \\
0 & \lambda & 0 & -\mu & 0 & 0 \\
-\lambda & 0 & \mu & 0 & 0 & 0
\end{array}\right) \quad \text { for } n_{13}=6,
\end{aligned}
$$

where $\lambda=\sqrt{(1+3 t) / 6}, \mu=\sqrt{(1-3 t) / 6}$. For a fixed $t \in\left[-\frac{1}{3}, \frac{1}{3}\right]$ let $N_{t}$ be the $N$-algebra of type I with $B_{t}$ as the matrix of the structure constants. Let $t, s \in\left[-\frac{1}{3}, \frac{1}{3}\right]$. Then it follows from Proposition 4.5 that $N_{t}$ is isomorphic to $N_{s}$ if and only if $t= \pm s$. It remains to determine the complex structures with respect to which $N_{t}\left(t \in\left[0, \frac{1}{3}\right]\right)$ is an $N$-algebra of type II. Let $J_{t}=\left(J_{t 13}, J_{t 23}\right)$ be a pair of the orthogonal matrices of degree $n_{13}$ and 2 , respectively.

Case I. Suppose $t \neq \frac{1}{3}$. Then $J_{t}$ satisfies (4.8) if and only if it is written as follows;

$$
J_{t 13}=\left\{\begin{array}{ll}
I\left(\varepsilon_{1}\right) \oplus I\left(\varepsilon_{1}\right) & \text { for } n_{13}=4 \\
I\left(\varepsilon_{1}\right) \oplus I\left(\varepsilon_{1}\right) \oplus I\left(\varepsilon_{2}\right) & \text { for } n_{13}=6
\end{array}, J_{t 23}=I\left(\varepsilon_{1}\right),\right.
$$

where $\varepsilon_{1}, \varepsilon_{2}= \pm 1$. We put

$$
T_{1}=\left(\begin{array}{ll}
1 & 0 \\
0 & \varepsilon_{1}
\end{array}\right), \quad T_{2}= \begin{cases}T_{1} \oplus T_{1} & \text { for } n_{13}=4 \\
T_{1} \oplus T_{1} \oplus\left(\begin{array}{ll}
1 & 0 \\
0 & \varepsilon_{2}
\end{array}\right) & \text { for } n_{13}=6 .\end{cases}
$$

Then, from the direct verification it follows that $T_{1} J_{t 23}=J_{23} T_{1}, T_{2} J_{t 13}=$ $J_{13} T_{2}$ and $\left(T_{1} \otimes T_{1}\right) B_{t}=B_{t} T_{2}$, which shows that $\left(J_{t 13}, J_{t 23}\right) \sim\left(J_{13}, J_{23}\right)$ (cf. Definition 4.7).

Case II. Suppose $t=\frac{1}{3}$. Then $J_{t}$ satisfies (4.8) if and only if $J_{t 23}=$ $I\left(\varepsilon_{1}\right)$ and 


$$
J_{t 13}= \begin{cases}I\left(\varepsilon_{1}\right) \oplus I\left(\varepsilon_{2}\right) & \text { for } n_{13}=4 \\ I\left(\varepsilon_{1}\right) \oplus J_{t 13}^{\prime} & \text { for } n_{13}=6,\end{cases}
$$

where $J_{t 13}^{\prime} \in O(4)$ and $J_{t 13}^{\prime 2}=-E_{4}$. There exists a matrix $T \in O(4)$ such that $T J_{t 13}^{\prime}=(I(1) \oplus I(1)) T$. Put

$$
T_{3}= \begin{cases}T_{1} \oplus\left(\begin{array}{ll}
1 & 0 \\
0 & \varepsilon_{2}
\end{array}\right) & \text { for } n_{13}=4 \\
T_{1} \oplus T & \text { for } n_{13}=6 .\end{cases}
$$

Then it can be seen that $T_{1} J_{t 23}=J_{23} T_{1}, T_{3} J_{t 13}=J_{13} T_{3}$ and $\left(T_{1} \otimes T_{1}\right) B_{1 / 3}=$ $B_{1 / 3} T_{3}$, which shows $\left(J_{t 13}, J_{t 23}\right) \sim\left(J_{13}, J_{23}\right)$.

Thus it follows from Lemma 4.8 that there exists a unique complex structure $J$ with respect to which $N_{t}$ is of type II for each $t \in\left[0, \frac{1}{3}\right]$.

Proposition 6.5. There exist two non-isomorphic $N$-algebras $\left(N, J^{(1)}\right)$ and $\left(N, J^{(2)}\right)$ corresponding to $\mathfrak{S}_{2}^{2}$ with $\left(n_{12}, n_{23}, n_{13}\right)=(2,4,4)$. They are isomorphic to each other as $N$-algebras of type $I$, but the complex structure $J^{(1)}$ and $J^{(2)}$ are not equivalent: the multiplication and the complex structures $J^{(1)}$ and $J^{(2)}$ are given by

$$
\begin{gathered}
\begin{cases}e_{12}^{1} e_{23}^{1}=\frac{1}{2} e_{13}^{2} & e_{12}^{2} e_{23}^{1}=\frac{1}{2} e_{13}^{1} \\
e_{12}^{1} e_{23}^{2}=-\frac{1}{2} e_{13}^{1} & e_{12}^{2} e_{23}^{2}=\frac{1}{2} e_{13}^{2} \\
e_{12}^{1} e_{23}^{3}=\frac{1}{2} e_{13}^{4} & e_{12}^{2} e_{23}^{3}=\frac{1}{2} e_{13}^{3} \\
e_{12}^{1} e_{23}^{4}=-\frac{1}{2} e_{13}^{3} & e_{12}^{2} e_{23}^{4}=\frac{1}{2} e_{13}^{4} .\end{cases} \\
J_{13}^{(1)}=J_{23}^{(1)}=I(1) \oplus I(1), \quad J_{13}^{(2)}=J_{23}^{(2)}=I(1) \oplus I(-1) .
\end{gathered}
$$

Proof. The Grammian $A_{t}$ of the skeleton $S$ in $\S 4$ with $\left(n_{12}, n_{23}, n_{13}\right)$ $=(2,4,4)$ is

$$
A_{t}=\left(\begin{array}{cc}
\frac{1}{4} E_{4} & -\tilde{A}_{t} \\
\tilde{A}_{t} & \frac{1}{4} E_{4}
\end{array}\right), \quad \tilde{A}_{t}=\left(\begin{array}{rrrr}
0 & -t_{1} & -t_{5} & -t_{4} \\
t_{1} & 0 & -t_{3} & -t_{6} \\
t_{5} & t_{3} & 0 & -t_{2} \\
t_{4} & t_{6} & t_{2} & 0
\end{array}\right),
$$

where $t=\left(t_{1}, \cdots, t_{6}\right)$. The characteristic polynomial of $A_{t}$ is given as follows,

$$
\operatorname{det}\left(x E_{8}-A_{t}\right)=\left\{(x-1 / 4)^{4}-(x-1 / 4)^{2} \sum_{1 \leq k \leq 6} t_{k}^{2}+\left(t_{1} t_{2}+t_{3} t_{4}-t_{5} t_{6}\right)^{2}\right\}^{2} .
$$


Considering (4.3) and the fact that rank $A_{t} \leq n_{13}=4$, we conclude that for each value of $t$ the eigenvalues of $A_{t}$ are $\frac{1}{2}$ and 0 both with multiplicity 4. Furthermore we have

$$
\left\{\begin{array}{l}
t_{1}=\varepsilon t_{2}, t_{3}=\varepsilon t_{4}, t_{5}=-\varepsilon t_{6}, \\
t_{1}^{2}+t_{3}^{2}+t_{5}^{2}=\left(\frac{1}{4}\right)^{2},
\end{array}\right.
$$

where $\varepsilon= \pm 1$. So Proposition 4.5 shows that there exists a unique $N$ algebra of type I corresponding to $S$ with $\left(n_{12}, n_{23}, n_{13}\right)=(2,4,4)$. To get the multiplication of $N$, take a special value of the parameter $t$ satisfying (6.2), e.g., $t=\left(\frac{1}{4}, \frac{1}{4}, 0 \ldots 0\right)$, and put

$$
B=\frac{1}{2}\left(\begin{array}{c}
\tilde{B} \\
E_{4}
\end{array}\right), \quad \tilde{B}=\left(\begin{array}{rrrr}
0 & 1 & 0 & 0 \\
-1 & 0 & 0 & 0 \\
0 & 0 & 0 & 1 \\
0 & 0 & -1 & 0
\end{array}\right) \text {. }
$$

Then $B$ is a solution of the equation (4.4) for $t=\left(\frac{1}{4}, \frac{1}{4}, 0 \ldots 0\right)$ and the matrix $B$ gives the multiplication (6.1). Let $J=\left(J_{13}, J_{23}\right)$ be a pair of the orthogonal matrices of degree 4. Then $J$ satisfies (4.8) if and only if $J_{13}=J_{23}, J_{13}^{2}=-E_{4}, J_{13} \tilde{B}=\tilde{B} J_{13}$. Since $\tilde{B}=I(-1) \oplus I(-1)$, there exists an orthogonal matrix $T$ of degree 4 such that

$$
T \tilde{B}=\tilde{B} T, T J_{i 3}^{t} T=I\left(\varepsilon_{1}\right) \oplus I\left(\varepsilon_{2}\right) \quad(i=1,2),
$$

where $\left(\varepsilon_{1}, \varepsilon_{2}\right)=(1,1),(1,-1)$ or $(-1,-1)$. Since $T \tilde{B}=\tilde{B} T$ implies $\left(E_{2} \otimes T\right) B=B T$, it follows that $J \sim J^{\left(\varepsilon_{1}, \varepsilon_{2}\right)}$, where $J^{\left(\varepsilon_{1}, \varepsilon_{2}\right)}=\left(I\left(\varepsilon_{1}\right) \oplus I\left(\varepsilon_{2}\right)\right.$, $I\left(\varepsilon_{1}\right) \oplus I\left(\varepsilon_{2}\right)$ ) (cf. Definition 4.7). Put

$$
T_{1}=\left(\begin{array}{rr}
-1 & 0 \\
0 & 1
\end{array}\right), \quad T_{2}=\left(-T_{1}\right) \oplus\left(-T_{1}\right) .
$$

Then we have $\left(T_{1} \otimes T_{2}\right) B=B T_{2}, T_{2}(I(1) \oplus I(1))=(I(-1) \oplus I(-1)) T_{2}$. Hence, by Definition $4.7, J^{(1,1)} \sim J^{(-1,-1)}$. Suppose $J^{(1,-1)} \sim J^{(1,1)}$. Then there exist three matrices $T_{3} \in O(2), T_{4} \in O(4)$ and $T_{5} \in O(4)$ such that

$$
T_{i}(I(1) \oplus I(-1))=(I(1) \oplus I(1)) T_{i} \quad(i=4,5)
$$

and

$$
\left(T_{3} \otimes T_{4}\right) B=B T_{5}
$$

Putting $T_{3}=\left(\begin{array}{ll}a & c \\ b & d\end{array}\right)$, the condition (6.4) is equivalent to 


$$
\tilde{B} T_{5}=a T_{4} \tilde{B}+c T_{4}, \quad T_{5}=b T_{4} \tilde{B}+d T_{4} .
$$

Using (6.3) and (6.5), it follows from the direct calculation that $T_{4}$ is not invertible, which is a contradiction. So we have two different $N$ algebras $\left(N, J^{(1)}\right)$ and $\left(N, J^{(2)}\right)$.

q.e.d.

Proposition 6.6. There exists a unique N-algebra whose diagram is $\widetilde{S}_{2}^{2}$ with $\left(n_{12}, n_{23}, n_{13}\right)=(3,2,4)$. The multiplication and the complex structure are as follows,

$$
\left\{\begin{array}{c}
e_{12}^{1} e_{23}^{1}=\sqrt{\frac{2}{7}} e_{13}^{3}, \quad e_{12}^{2} e_{23}^{1}=-\sqrt{\frac{2}{7}} e_{13}^{4}, \quad e_{12}^{3} e_{23}^{1}=\sqrt{\frac{2}{7}} e_{13}^{1} \\
e_{12}^{1} e_{23}^{2}=\sqrt{\frac{2}{7}} e_{13}^{4}, \quad e_{12}^{2} e_{23}^{2}=\sqrt{\frac{2}{7}} e_{13}^{3}, \quad e_{12}^{3} e_{23}^{2}=\sqrt{\frac{2}{7}} e_{13}^{2} \\
J_{13}=I(1) \oplus I(1), \quad J_{23}=I(1) .
\end{array}\right.
$$

Proof. The Grammian $A_{t}$ of the skeleton $S$ in $\S 4$ with $\left(n_{12}, n_{23}, n_{13}\right)$ $=(3,2,4)$ contains three parameters $t=\left(t_{1}, t_{2}, t_{3}\right)$ and is represented as

$$
A_{t}=\tilde{A}_{t} \otimes\left(\begin{array}{rr}
0 & -1 \\
1 & 0
\end{array}\right)+\frac{2}{7} E_{6}, \quad \tilde{A}_{t}=\left(\begin{array}{rrr}
0 & -t_{1} & -t_{2} \\
t_{1} & 0 & -t_{3} \\
t_{2} & t_{3} & 0
\end{array}\right)
$$

The eigenvalues of $A_{t}$ are $\frac{2}{7}$ and $\frac{2}{7} \pm \sqrt{t_{1}^{2}+t_{2}^{2}+t_{3}^{2}}$ with multiplicity 2, respectively. Since rank $A_{t} \leq n_{13}=4$, we get $t_{1}^{2}+t_{2}^{2}+t_{3}^{2}=\left(\frac{2}{7}\right)^{2}$ and so, for each value of the parameter $t$ the eigenvalues of $A_{t}$ are $\frac{4}{7}, \frac{2}{7}$ and 0 with multiplicity 2 , respectively. So the corresponding $N$-algebras of type I are isomorphic to each other (cf. Proposition 4.5). Put

$$
B=\sqrt{\frac{2}{7}}\left(\begin{array}{rrrr}
0 & 0 & 1 & 0 \\
0 & 0 & 0 & 1 \\
0 & 0 & 0 & -1 \\
0 & 0 & 1 & 0 \\
1 & 0 & 0 & 0 \\
0 & 1 & 0 & 0
\end{array}\right)
$$

Then $B$ is a solution of the equation (4.4) for $t=\left(\frac{2}{7}, 0,0\right)$ and $B$ gives the multiplication (6.6). Let $J=\left(J_{13}, J_{23}\right)$ be a pair of the orthogonal matrices of degree 4 and 2, respectively. Then $J$ satisfies (4.8) if and only if $J$ is represented as follows,

$$
J_{13}=I(\varepsilon) \oplus I(\varepsilon), J_{23}=I(\varepsilon), \quad \varepsilon= \pm 1 .
$$


We write $J^{(\varepsilon)}=\left(J_{13}^{(\varepsilon)}, J_{23}^{(\varepsilon)}\right)$ instead of $J=\left(J_{13}, J_{23}\right)$ in accordance with $\varepsilon=$ \pm 1 . Put

$$
T_{1}=\left(\begin{array}{lll}
1 & & 0 \\
& -1 & \\
0 & & 1
\end{array}\right), \quad T_{2}=\left(\begin{array}{rr}
1 & 0 \\
0 & -1
\end{array}\right), \quad T_{3}=T_{2} \oplus T_{2}
$$

Then we have $J_{13}^{(-1)} T_{3}=T_{3} J_{13}^{(1)}, J_{23}^{(-1)} T_{2}=T_{2} J_{23}^{(1)},\left(T_{1} \otimes T_{2}\right) B=B T_{3}$, which implies $J^{(1)} \sim J^{(-1)}$.

q.e.d.

The following proposition can be proved by the similar methods as in Lemma 4.9, Proposition 6.2 and 6.3, since $n_{12}, n_{24} \leq 2$ for $\mathfrak{S}_{3}^{2}$. Thus we omit the proof.

Proposition 6.7. There exists a unique N-algebra whose diagram is $\mathfrak{\Im}_{3}^{2}\left(\right.$ resp. $\left.\widetilde{\subseteq}_{3}^{6}\right)$.

Proposition 6.8. There exists a unique N-algebra whose diagram is $\mathfrak{S}_{3}^{5}$. Furthermore the multiplication and the complex structure are as follows,

$$
\left\{\begin{array}{l}
e_{13}^{1} e_{34}^{k}=\frac{1}{\sqrt{3}} e_{14}^{k}, e_{23}^{1} e_{34}^{k}=\frac{1}{\sqrt{3}} e_{24}^{k} \quad(k=1,2), \\
J_{14}=J_{24}=J_{34}=I(1) .
\end{array}\right.
$$

Proof. Let $N=N_{13}+N_{34}+N_{14}+N_{23}+N_{24}$ be an orthogonal direct sum of the euclidean vector spaces $N_{i j}$ of dimension $n_{i j}$ with an inner product $\langle$,$\rangle , where \left(n_{13}, n_{34}, n_{14}, n_{23}, n_{24}\right)=(1,2,2,1,2)$. We define the multiplication and the complex structure $j$ in $N$ by (6.7). Then it is easy to see that with this structure, $(N,\langle\rangle, j$,$) is an N$-algebra corresponding to $\mathfrak{S}_{3}^{5}$. Let $\left(N^{\prime},\langle,\rangle^{\prime}, j^{\prime}\right)$ be another $N$-algebra whose diagram is $\mathfrak{S}_{3}^{5}$. Then, from Proposition 6.2 it follows that there exists a gradepreserving linear isometry $f$ of $N_{13}+N_{34}+N_{14}$ onto $N_{13}^{\prime}+N_{34}^{\prime}+N_{14}^{\prime}$ such that $f(x y)=f(x) f(y)$ for $x \in N_{13}$ and $y \in N_{34}$ and that $f \circ j=j^{\prime} \circ f$ on $N_{34}$ $+N_{14}$. We will extend $f$ to an isomorphism of $N$ onto $N^{\prime}$. Let $h$ be a linear isometry of $N_{23}$ onto $N_{23}^{\prime}$, and let $L_{e_{23}^{1}}$ and $L_{h\left(e_{23}^{1}\right)}$ be the left multiplication by $e_{23}^{1}$ and $h\left(e_{23}^{1}\right)$, respectively. We define a map $g$ of $N$ onto $N^{\prime}$ as

$$
g= \begin{cases}h & \text { on } N_{23}, \\ L_{h\left(e_{23}^{1}\right)} \circ f \circ L_{e_{23}^{-1}}^{-1} & \text { on } N_{24}, \\ f & \text { on } N_{13}+N_{34}+N_{14}\end{cases}
$$


Then, by (N4) in $\S 1 g$ is a grade-preserving linear isometry. Since $L_{e_{23}^{1}} \circ j=j \circ L_{e_{23}^{1}}$ and $L_{h\left(e_{23}^{1}\right)} \circ j^{\prime}=j^{\prime} \circ L_{h\left(e_{23}^{\frac{1}{1}}\right)}$ (cf. Definition 2.3), we have $g \circ j$ $=j^{\prime} \circ g$ on $N_{14}+N_{24}+N_{34}$. To show that $g$ is a homomorphism, it is enough to verify that $g(x y)=g(x) g(y)$ for $x \in N_{23}$ and $y \in N_{34}$. We can assume that $x=e_{23}^{1}$. Then $g\left(e_{23}^{1} y\right)=\left(L_{h\left(e_{23}^{1}\right)} \circ f \circ L_{e_{23}^{1}}^{-1}\right)\left(e_{23}^{1} y\right)=h\left(e_{23}^{1}\right) f(y)=$ $g\left(e_{23}^{1}\right) g(y)$. We have thus proved that $N$ is isomorphic to $N^{\prime}$. q.e.d.

By the analogous way as in the above proposition, we get

Proposition 6.9. There exists a unique N-algebra whose diagram is $\mathfrak{S}_{3}^{7}$. The multiplication and the complex structure are given as follows,

$$
\begin{gathered}
e_{12}^{1} e_{23}^{1}=\frac{1}{\sqrt{3}} e_{13}^{1}, e_{12}^{1} e_{24}^{k}=\frac{1}{\sqrt{3}} e_{14}^{k} \quad(k=1,2) . \\
J_{14}=J_{24}=I(1) .
\end{gathered}
$$

We have thus showed that only to the skeleton $\mathfrak{S}_{2}^{2}$ there correspond several non-isomorphic $N$-algebras, and a skeleton isomorphic to $\mathfrak{S}_{2}^{2}$ is $\widetilde{S}_{2}^{2}$ itself. Hence, in view of the above propositions and Remark 4.10, we have solved the problem III (§3) for $N$-algebras of type II.

\section{§ 7. Final Results}

7.1. Summing up results in $\S 5$ and $\S 6$, we get the following

THEOREM 7.1. (1) There exists a one-to-one correspondence between the set of (holomorphic) equivalence classes of all irreducible homogeneous Siegel domains of type I up to dimension 10 and the set of all the skeletons in Proposition 3.5.

(2) (i) To each of the skeletons in Proposition 3.6 except $\widetilde{\subseteq}_{2}^{2}$ with $\left(n_{12}, n_{23}, n_{13}\right)=(2,2,4),(2,2,6)$ or $(2,4,4)$, there corresponds one and only one irreducible homogeneous Siegel domain of type II of dimension $\leq 8$;

(ii) to the skeleton $\mathfrak{S}_{2}^{2}$ with $\left(n_{12}, n_{23}, n_{13}\right)=(2,2,4)$ or $(2,2,6)$ there corresponds a one-parameter family of non-equivalent irreducible homogeneous Siegel domains of type II of dimension 7 or 8;

(iii) to the skeleton $\mathfrak{S}_{2}^{2}$ with $\left(n_{12}, n_{23}, n_{13}\right)=(2,4,4)$ there correspond two non-equivalent irreducible homogeneous Siegel domains of type II of dimension 8; the domains in (i)-(iii) exhaust all irreducible homogeneous Siegel domains of type II of dimension $\leq 8$.

By the above theorem, we can count the numbers of all irreducible 
homogeneous Siegel domains of type I (resp. type II) up to dimension 10 (resp. 8). And we have

THEOREM 7.2. Let $\Psi_{\mathrm{I}}(n)$ (resp. $\Psi_{\mathrm{II}}(n)$ ) denote the number of irreducible homogeneous bounded domains of dimension $n$ which are realized as Siegel domains of type $I$ (resp. type $I I)$. Then $\Psi_{I}(n)$ and $\Psi_{I I}(n)$ are given as follows;

\begin{tabular}{|l|cccccccccc|}
\hline$n$ & 1 & 2 & 3 & 4 & 5 & 6 & 7 & 8 & 9 & 10 \\
\hline$\Psi_{\mathrm{I}}(n)$ & 1 & 0 & 1 & 1 & 3 & 4 & 9 & 16 & 34 & 66 \\
\hline$\Psi_{\mathrm{II}}(n)$ & 0 & 1 & 1 & 2 & 4 & 8 & $15+\infty^{1}$ & $34+\infty^{1}$ & & \\
\hline
\end{tabular}

where $\infty^{1}$ denotes a one-parameter family of the domains.

7.2. We will give here the explicit forms of all irreducible homogeneous convex cones up to dimension 7 . These forms are obtained by using the multiplications of $N$-algebras described in $\S 5$ and a result of Vinberg [8]. In what follows we will use the following notations:

$V \quad$ A homogeneous convex cone in a real vector space.

$H(n: R) \quad$ The vector space of all real symmetric matrices of degree $n$.

$\boldsymbol{R}^{+} \quad$ The cone of all positive real numbers.

$H^{+}(n: \boldsymbol{R}) \quad$ The cone of all positive definite matrices in $H(n: \boldsymbol{R})$.

$C(n) \quad$ The circular cone of dimension $n$, that is, the set $\left\{\left(x_{1} \cdots x_{n}\right) \in \boldsymbol{R}^{n} ; x_{1} x_{2}-x_{3}^{2}-\cdots-x_{n}^{2}>0, x_{1}>0\right\}$.

$b, c, d, a_{i}, c_{i}, d_{i}(i=1,2, \cdots)$ Real variables.

$z, z_{i}(i=1,2, \cdots)$ Complex variables.

In the following list, the homogeneous convex cones (1.1), (1.2), (1.3), (1.4) and (1.5) are well known (for the last two, see Vinberg [8]), while others are new; the only cones (1.1),(1.2),(1.3) are self-dual.

1
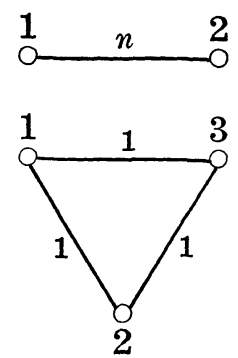

$$
V=R^{+}
$$$$
V=C(n+2)
$$$$
V=H^{+}(3: R)
$$

$\operatorname{dim} V=1$

$\operatorname{dim} V=n+2$.

$\operatorname{dim} V=6$. 
(1.4)

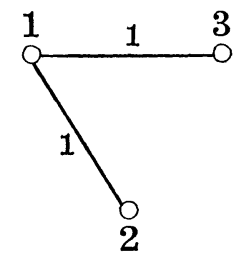

(1.5)

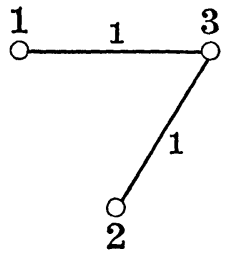

(1.6)

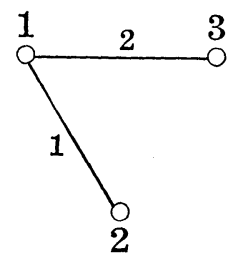

$$
V=\left\{\left(\begin{array}{lll}
a_{1} & b & z \\
b & a_{2} & 0 \\
\bar{z} & 0 & a_{3}
\end{array}\right)>0\right\}, \quad \operatorname{dim} V=6
$$

(1.7)

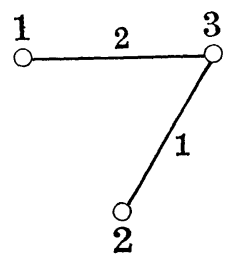

$$
\begin{gathered}
V=\left\{\left(\begin{array}{lll}
a_{1} & 0 & z \\
0 & a_{2} & b \\
\bar{z} & b & a_{3}
\end{array}\right) ;\left(\begin{array}{ll}
a_{1} & z \\
\bar{z} & a_{3}
\end{array}\right)>0,\left(\begin{array}{ll}
a_{2} & b \\
b & a_{3}
\end{array}\right)>0\right\}, \\
\operatorname{dim} V=6 .
\end{gathered}
$$

(1.8)

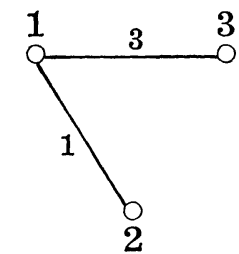

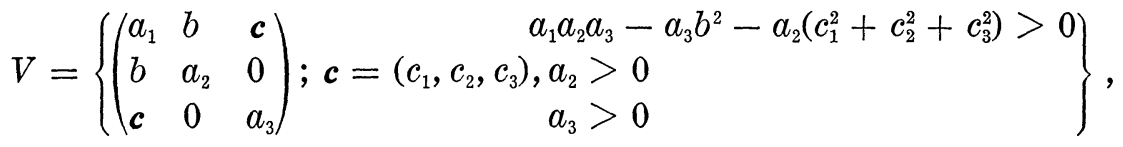

$\operatorname{dim} V=7$.

(1.9)

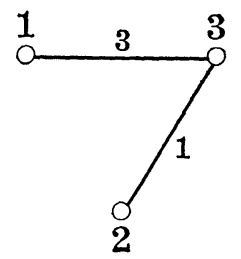




$$
V=\left\{\left(\begin{array}{ccc}
a_{1} & 0 & c \\
0 & a_{2} & b \\
c & b & a_{3}
\end{array}\right) ; c=\left(c_{1}, c_{2}, c_{3}\right), \begin{array}{c}
a_{3} a_{1}-\left(c_{1}^{2}+c_{2}^{2}+c_{3}^{2}\right)>0 \\
a_{3} a_{2}-b^{2}>0 \\
a_{3}>0 \\
\operatorname{dim} V=7
\end{array}\right\}
$$

(1.10)

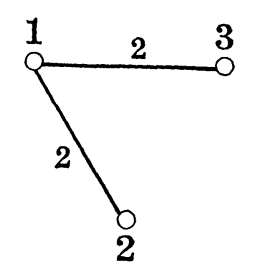

$$
V=\left\{\left(\begin{array}{lll}
a_{1} & z_{1} & z_{2} \\
\bar{z}_{1} & a_{2} & 0 \\
\bar{z}_{2} & 0 & a_{3}
\end{array}\right)>0\right\}, \quad \operatorname{dim} V=7
$$

$(1.11)$

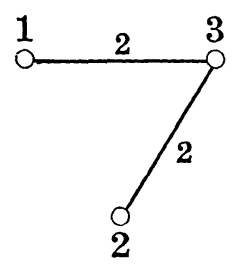

$$
\begin{gathered}
V=\left\{\left(\begin{array}{ccc}
a_{1} & 0 & z_{1} \\
0 & a_{2} & z_{2} \\
\bar{z}_{1} & \bar{z}_{2} & a_{3}
\end{array}\right) ;\left(\begin{array}{cc}
a_{1} & z_{1} \\
\bar{z}_{1} & a_{3}
\end{array}\right)>0,\left(\begin{array}{cc}
a_{2} & z_{2} \\
\bar{z}_{2} & a_{3}
\end{array}\right)>0\right\}, \\
\operatorname{dim} V=7 .
\end{gathered}
$$

(1.12)

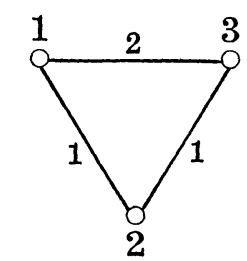

$$
V=\left\{\left(\begin{array}{lll}
a_{1} & b & \boldsymbol{d} \\
b & a_{2} & c \\
\boldsymbol{d} & c & a_{3}
\end{array}\right) ; \boldsymbol{d}=\left(d_{1}, d_{2}\right), \begin{array}{l}
\left(a_{3} a_{1}-d_{1}^{2}-d_{2}^{2}\right)\left(a_{3} a_{2}-c^{2}\right)-\left(c_{3} b-c d_{1}\right)^{2}>0 \\
a_{3}>0
\end{array}\right\},
$$

$\operatorname{dim} V=7$.

(1.13)

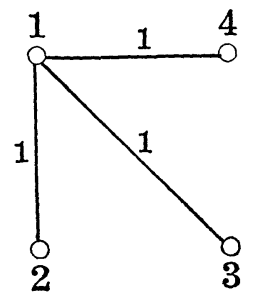

$$
V=\left\{\left(\begin{array}{llll}
a_{1} & b & c & d \\
b & a_{2} & 0 & 0 \\
c & 0 & a_{3} & 0 \\
d & 0 & 0 & a_{4}
\end{array}\right\}>0\right\}, \quad \operatorname{dim} V=7
$$

(1.14)

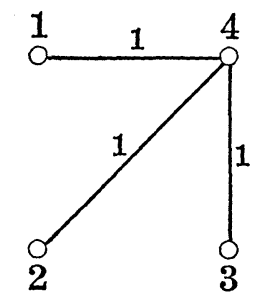




$$
\begin{gathered}
V=\left\{\left(\begin{array}{llll}
a_{1} & 0 & 0 & d \\
0 & a_{2} & 0 & c \\
0 & 0 & a_{3} & b \\
d & c & b & a_{4}
\end{array}\right) ;\left(\begin{array}{ll}
a_{1} & d \\
d & a_{4}
\end{array}\right)>0,\left(\begin{array}{ll}
a_{2} & c \\
c & a_{4}
\end{array}\right)>0,\left(\begin{array}{ll}
a_{3} & b \\
b & a_{4}
\end{array}\right)>0\right. \\
\operatorname{dim} V=7
\end{gathered}
$$

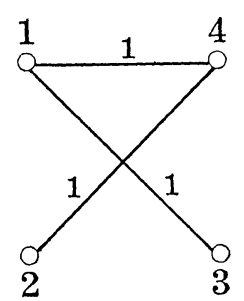

$$
\begin{gathered}
\left.V=\left\{\begin{array}{cccc}
a_{1} & 0 & b & d \\
0 & a_{2} & 0 & c \\
b & 0 & a_{3} & 0 \\
d & c & 0 & a_{4}
\end{array}\right) ;\left(\begin{array}{lll}
a_{1} & b & d \\
b & a_{3} & 0 \\
d & 0 & a_{4}
\end{array}\right)>0,\left(\begin{array}{ll}
a_{2} & c \\
c & a_{4}
\end{array}\right)>0\right\}, \\
\operatorname{dim} V=7 .
\end{gathered}
$$

7.3. We will give here the explicit forms of all irreducible homogeneous Siegel domains $D(V, F)$ of type II up to dimension 8 . These forms are obtained by using the results in $\S 6$ and a result of Takeuchi [7]. As we mentioned in 3.4, the diagram of the cone $V$ is obtained from the diagram of $D(V, F)$ by eliminating the black vertex and all line segments starting from it. And by the assumption for $D(V, F)$ the cone $V$ is irreducible and $\operatorname{dim} V \leq 7$. Hence, one can find the explicit form of the cone $V$ by the list of 7.2. So we will give only the $V$-hermitian form $F$.

In the following list, the domains (2.1), (2.2), (2.3), (2.4), (2.5), (2.7.a), (2.7.b), (2.17) with $n=1$ and (2.18) are found in Pjateckii-Sapiro [5], [6] (For (2.5), (2.6) see also [7]), while others are new. The domain (2.5) is different from a domain of Pjateckii-Sapiro (cf. [6] p. 28) in the form, but it can be seen that they are linearly equivalent. The domains (2.7.a) and (2.7.b) correspond to the $N$-algebras in Proposition 6.5 with the complex structures $J^{(1)}$ and $J^{(2)}$, respectively. The only domains (2.1), (2.4), (2.7.a) are symmetric.

$$
\begin{array}{lll}
\stackrel{1}{1} 2 m \quad 2 & 1 \leq m \leq 7, \quad F(u, v)=\sum_{k=1}^{m} u_{k} \bar{v}_{k}, \\
& \operatorname{dim} D(V, F)=m+1 .
\end{array}
$$




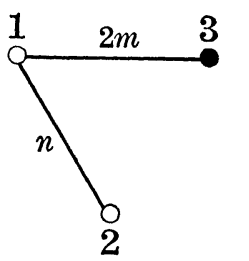

$$
\begin{aligned}
& 2 \leq n+m \leq 6, \\
& F(u, v)=\left(\sum_{k=1}^{m} u_{k} \bar{v}_{k}, 0, \cdots, 0\right) \in C^{n+2}, \\
& \operatorname{dim} D(V, F)=n+m+2 .
\end{aligned}
$$

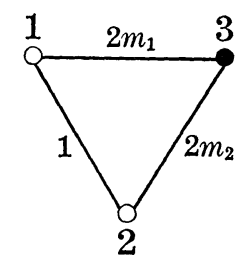

$$
\begin{aligned}
& \left(m_{1}, m_{2}\right)=(1,1),(2,1),(3,1),(4,1),(2,2),(3,2), \\
& \operatorname{dim} D(V, F)=m_{1}+m_{2}+3 .
\end{aligned}
$$

$$
\begin{aligned}
& F(u, v)=\left(\begin{array}{cc}
\sum_{k=1}^{m_{1}} u_{k} \bar{v}_{k} & \frac{1}{2} \sum_{k=1}^{m_{2}}\left(u_{k} \bar{v}_{m_{1}+k}+u_{m_{1}+k} \bar{v}_{k}\right) \\
\frac{1}{2} \sum_{k=1}^{m_{2}}\left(u_{k} \bar{v}_{m_{1}+k}+u_{m_{1}+k} \bar{v}_{k}\right) & \sum_{k=1}^{m_{2}} u_{m_{1}+k} \bar{v}_{m_{1}+k}
\end{array}\right), \\
& \text { where } u=\left(u_{1}, \cdots, u_{m_{1}+m_{2}}\right), v=\left(v_{1}, \cdots, v_{m_{1}+m_{2}}\right) \text {. }
\end{aligned}
$$

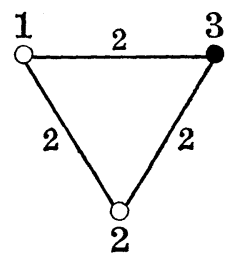

$$
F(u, v)=\left(\begin{array}{ll}
u_{1} \bar{v}_{1} & u_{1} \bar{v}_{2} \\
u_{2} \bar{v}_{1} & u_{2} \bar{v}_{2}
\end{array}\right), \quad \operatorname{dim} D(V, F)=6
$$

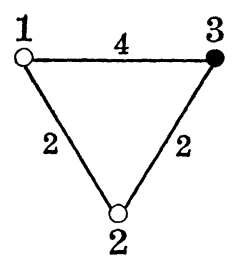

$$
\begin{aligned}
& F_{\theta}(u, v)=\left(\begin{array}{cc}
u_{1} \bar{v}_{1}+u_{2} \bar{v}_{2} & \operatorname{Cos} \theta \cdot u_{1} \bar{v}_{3}+\operatorname{Sin} \theta \cdot u_{3} \bar{v}_{2} \\
\operatorname{Cos} \theta \cdot u_{3} \bar{v}_{1}+\operatorname{Sin} \theta \cdot u_{2} \bar{v}_{3} & u_{3} \bar{v}_{3}
\end{array}\right), \\
& \text { where } 0 \leq \theta \leq \pi / 4, \operatorname{dim} D\left(V, F_{\theta}\right)=7 .
\end{aligned}
$$

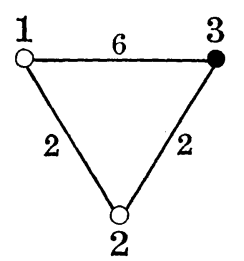

$$
\begin{gathered}
F_{\theta}(u, v)=\left(\begin{array}{cc}
u_{1} \bar{v}_{1}+u_{2} \bar{v}_{2}+u_{3} \bar{v}_{3} & \operatorname{Cos} \theta \cdot u_{1} \bar{v}_{4}+\operatorname{Sin} \theta \cdot u_{4} \bar{v}_{2} \\
\operatorname{Cos} \theta \cdot u_{4} \bar{v}_{1}+\operatorname{Sin} \theta \cdot u_{2} \bar{v}_{4} & u_{4} \bar{v}_{4}
\end{array}\right), \\
\text { where } 0 \leq \theta \leq \pi / 4, \operatorname{dim} D\left(V, F_{\theta}\right)=8 .
\end{gathered}
$$


(2.7.a)

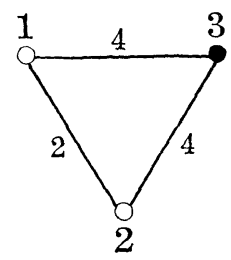

$(2.7 . b)$

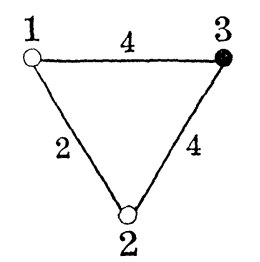

$(2.8)$

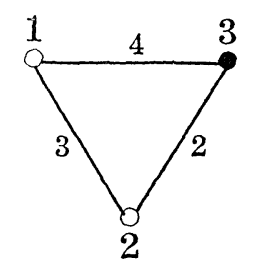

(2.9)

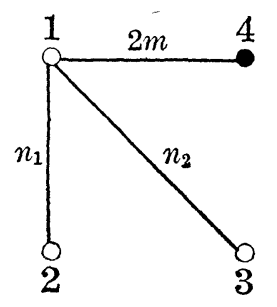

$(2.10)$

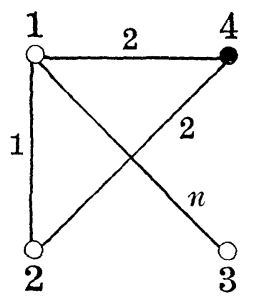

(2.11)

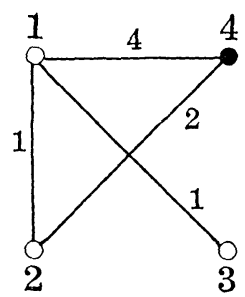

$F(u, v)=\left(\begin{array}{ll}u_{1} \bar{v}_{1}+u_{2} \bar{v}_{2} & u_{3} \bar{v}_{1}+u_{4} \bar{v}_{2} \\ u_{1} \bar{v}_{3}+u_{2} \bar{v}_{4} & u_{3} \bar{v}_{3}+u_{4} \bar{v}_{4}\end{array}\right)$, $\operatorname{dim} D(V, F)=8$.

$F(u, v)=\left(\begin{array}{ll}u_{1} \bar{v}_{1}+u_{2} \bar{v}_{2} & u_{3} \bar{v}_{1}+u_{2} \bar{v}_{4} \\ u_{1} \bar{v}_{3}+u_{4} \bar{v}_{2} & u_{3} \bar{v}_{3}+u_{4} \bar{v}_{4}\end{array}\right)$, $\operatorname{dim} D(V, F)=8$.

$F(u, v)=\left(u_{1} \bar{v}_{1}+u_{2} \bar{v}_{2}, u_{3} \bar{v}_{3}, \frac{1}{2}\left(u_{2} \bar{v}_{3}+u_{3} \bar{v}_{2}\right)\right.$, $\left.\frac{2}{2}\left(u_{2} \bar{v}_{3}-u_{3} \bar{v}_{2}\right), \frac{1}{2}\left(u_{1} \bar{v}_{3}+u_{3} \bar{v}_{1}\right)\right)$, $\operatorname{dim} D(V, F)=8$.

$\left(n_{1}, n_{2}, m\right)=(1,1,1),(1,1,2),(1,1,3)$, $(1,2,1),(1,2,2),(1,3,1)$, $(2,2,1)$.

$F(u, v)=\left(\begin{array}{ccc}\sum_{k=1}^{m} u_{k} \bar{v}_{k} & 0 & 0 \\ 0 & 0 & 0 \\ 0 & 0 & 0\end{array}\right)$ $\operatorname{dim} D(V, F)=n_{1}+n_{2}+m+3$.

$n=1$ or 2 ,

$F(u, v)=\left(\begin{array}{ccc}u_{1} \bar{v}_{1} & \frac{1}{2}\left(u_{2} \bar{v}_{1}+u_{1} \bar{v}_{2}\right) & 0 \\ \frac{1}{2}\left(u_{2} \bar{v}_{1}+u_{1} \bar{v}_{2}\right) & u_{2} \bar{v}_{2} & 0 \\ 0 & 0 & 0\end{array}\right)$, $\operatorname{dim} D(V, F)=n+6$.

$F(u, v)=\left(\begin{array}{ccc}u_{1} \bar{v}_{1}+u_{2} \bar{v}_{2} & \frac{1}{2}\left(u_{1} \bar{v}_{3}+u_{3} \bar{v}_{1}\right) & 0 \\ \frac{1}{2}\left(u_{1} \bar{v}_{3}+u_{3} \bar{v}_{1}\right) & u_{3} \bar{v}_{3} & 0 \\ 0 & 0 & 0\end{array}\right)$, $\operatorname{dim} D(V, F)=8$. 
(2.12)

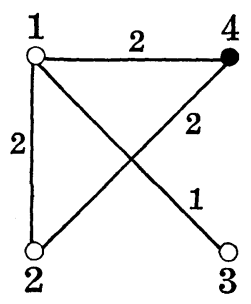

(2.13)

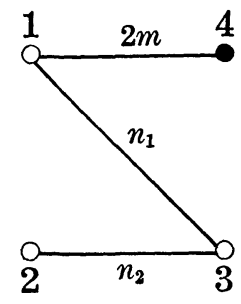

(2.14)

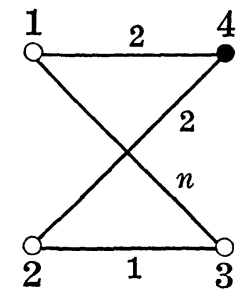

$(2.15)$
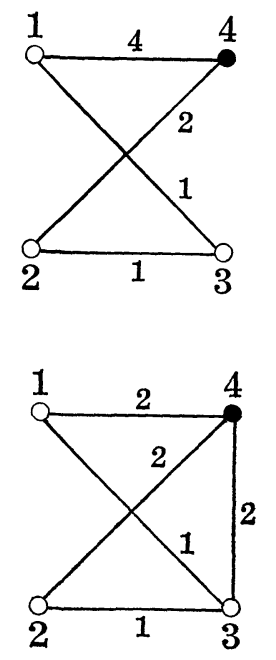

$F(u, v)=\left(\begin{array}{ccc}u_{1} \bar{v}_{1} & u_{1} \bar{v}_{2} & 0 \\ u_{2} \bar{v}_{1} & u_{2} \bar{v}_{2} & 0 \\ 0 & 0 & 0\end{array}\right), \quad \operatorname{dim} D(V, F)=8$

$\left(n_{1}, n_{2}, m\right)=(1,1,1),(1,1,2),(1,1,3),(2,1,1)$, $(2,1,2),(3,1,1),(2,2,1),(1,2,1)$, $(1,2,2),(1,3,1)$,

$F(u, v)=\left(\begin{array}{ccc}\sum_{k=1}^{m} u_{k} \bar{v}_{k} & 0 & 0 \\ 0 & 0 & 0 \\ 0 & 0 & 0\end{array}\right)$,

$\operatorname{dim} D(V, F)=n_{1}+n_{2}+m+3$.

$n=1$ or 2 ,

$F(u, v)=\left(\begin{array}{ccc}n_{1} \bar{v}_{1} & 0 & 0 \\ 0 & u_{2} \bar{v}_{2} & 0 \\ 0 & 0 & 0\end{array}\right)$,

$\operatorname{dim} D(V, F)=n+6$.

$F(u, v)=\left(\begin{array}{ccc}u_{1} \bar{v}_{1}+u_{2} \bar{v}_{2} & 0 & 0 \\ 0 & u_{3} \bar{v}_{3} & 0 \\ 0 & 0 & 0\end{array}\right)$

$\operatorname{dim} D(V, F)=8$.

$$
\begin{aligned}
& F(u, v)= \\
& \left(\begin{array}{ccc}
u_{1} \bar{v}_{1} & 0 & \frac{1}{2}\left(u_{3} \bar{v}_{1}+u_{1} \bar{v}_{3}\right) \\
0 & u_{2} \bar{v}_{2} & \frac{1}{2}\left(u_{3} \bar{v}_{2}+u_{2} \bar{v}_{3}\right) \\
\frac{1}{2}\left(u_{3} \bar{v}_{1}+u_{1} \bar{v}_{3}\right) & \frac{1}{2}\left(u_{3} \bar{v}_{2}+u_{2} \bar{v}_{3}\right) & u_{3} \bar{v}_{3}
\end{array}\right),
\end{aligned}
$$

$\operatorname{dim} D(V, F)=8$. 
(2.17)

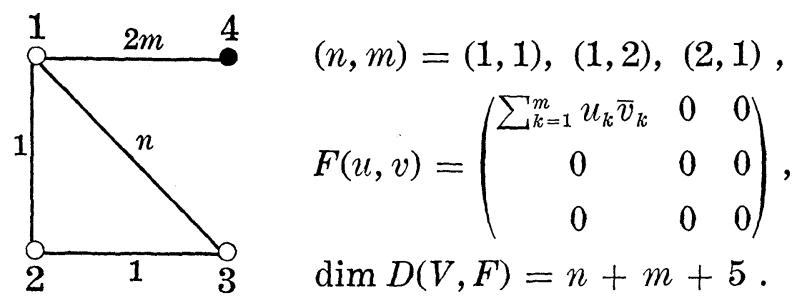

(2.18)

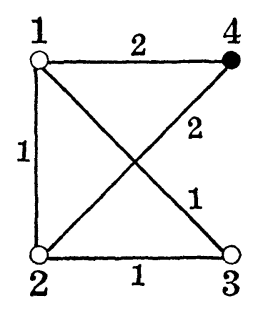

$$
F(u, v)=\left(\begin{array}{ccc}
u_{1} \bar{v}_{1} & \frac{1}{2}\left(u_{2} \bar{v}_{1}+u_{1} \bar{v}_{2}\right) & 0 \\
\frac{1}{2}\left(u_{2} \bar{v}_{1}+u_{1} \bar{v}_{2}\right) & u_{2} \bar{v}_{2} & 0 \\
0 & 0 & 0
\end{array}\right) \text {, }
$$

$\operatorname{dim} D(\dot{V}, F)=8$.

(2.19)

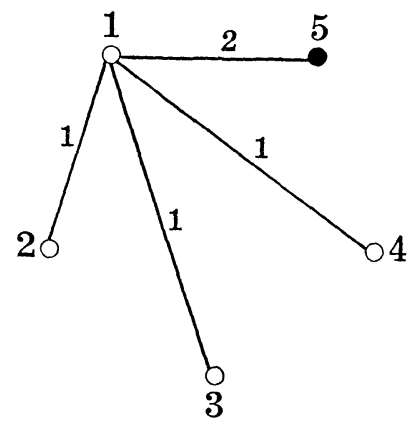

$$
\begin{aligned}
& F(u, v)=\left(\begin{array}{cccc}
u \bar{v} & 0 & 0 & 0 \\
0 & 0 & 0 & 0 \\
0 & 0 & 0 & 0 \\
0 & 0 & 0 & 0
\end{array}\right), \\
& \operatorname{dim} D(V, F)=8 .
\end{aligned}
$$

$(2.20)$

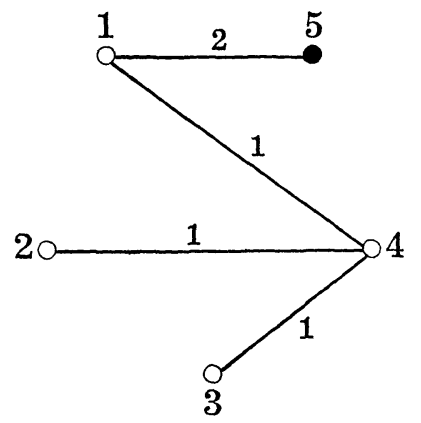

$F(u, v)=\left(\begin{array}{cccc}u \bar{v} & 0 & 0 & 0 \\ 0 & 0 & 0 & 0 \\ 0 & 0 & 0 & 0 \\ 0 & 0 & 0 & 0\end{array}\right)$,

$\operatorname{dim} D(V, F)=8$. 


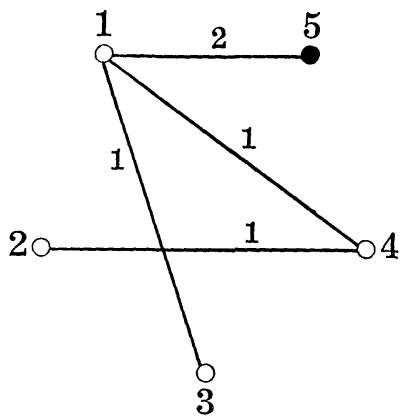

$$
F(u, v)=\left(\begin{array}{cccc}
u \bar{v} & 0 & 0 & 0 \\
0 & 0 & 0 & 0 \\
0 & 0 & 0 & 0 \\
0 & 0 & 0 & 0
\end{array}\right),
$$

$\operatorname{dim} D(V, F)=8$.

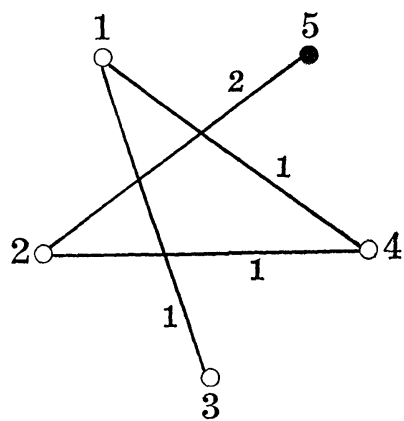

$$
\begin{aligned}
& F(u, v)=\left(\begin{array}{cccc}
0 & 0 & 0 & 0 \\
0 & u \bar{v} & 0 & 0 \\
0 & 0 & 0 & 0 \\
0 & 0 & 0 & 0
\end{array}\right), \\
& \operatorname{dim} D(V, F)=8 .
\end{aligned}
$$

\section{BIBLIOGRAPHY}

[1] H. Asano, On the irreducibility of homogeneous convex cones, J. Fac. Sci. Univ. Tokyo, 15 (1968), 201-208.

[2] F. R. Gantmacher, The theory of matrices, Vol. 1, Chelsea, New York, 1959.

[ 3 ] S. Kaneyuki, On the automorphism groups of homogeneous bounded domains, J. Fac. Sci. Univ. Tokyo 14 (1967), 89-130.

[4] W. Kaup, Y. Matsushima and T. Ochiai, On the automorphisms and equivalences of generalized Siegel domains, Amer. J. Math., 92 (1970), 475-498.

[5] I. I. Pjateckii-Sapiro, Geometry of classical domains and theory of automorphic functions, Fizmatgiz, Moscow, 1961, French translation, Paris, 1966.

[ 6 ] I. I. Pjateckii-Sapiro, Automorphic functions and the geometry of classical domains, Gordon and Breach, New York, 1969.

[ 7 ] M. Takeuchi, On infinitesimal affine automorphisms of Siegel domains, Proc. Japan Acad., 45 (1969), 590-594.

[ 8 ] E. B. Vinberg, The theory of convex homogeneous cones, Trudy Moskva Math. Obsc., 12 (1963), 303-358. Trans. Moscow Math. Soc., 12 (1963), 340-403.

[9] E. B. Vinberg, The structure of the group of automorphisms of a homogeneous convex cone, Trudy Moskva Math. Obsc., 13 (1965), 56-83. Trans. Moscow Math. Soc., 13 (1965), 63-93.

[10] E. B. Vinberg, S. G. Gindikin and I. I. Pjateckii-Sapiro, On classification and canonical realization of complex bounded homogeneous domains, Trudy Moskva Math. Obsc., 12 (1963), 359-388. Trans. Moscow Math. Soc., 12 (1963), 404-437.

Department of Mathematics

Nagoya University 